\title{
TrkB agonists prevent postischemic emergence of refractory neonatal seizures in mice
}

\author{
Pavel A. Kipnis, ${ }^{1}$ Brennan J. Sullivan, ${ }^{1}$ Brandon M. Carter, ${ }^{1}$ and Shilpa D. Kadam ${ }^{1,2}$ \\ ${ }^{1}$ Neuroscience Laboratory, Hugo W. Moser Research Institute, Kennedy Krieger Institute, Baltimore, Maryland, USA \\ 2Department of Neurology and Neurosurgery, Johns Hopkins University School of Medicine, Baltimore, Maryland, USA.
}

Refractory neonatal seizures do not respond to first-line antiseizure medications like phenobarbital (PB), a positive allosteric modulator for $\mathrm{CABA}_{A}$ receptors. $G A B A_{A}$ receptor-mediated inhibition is dependent upon electroneutral cation-chloride transporter KCC2, which mediates neuronal chloride extrusion and its age-dependent increase and postnatally shifts CABAergic signaling from depolarizing to hyperpolarizing. Brain-derived neurotropic factor-tyrosine receptor kinase $B$ activation (BDNF-TrkB activation) after excitotoxic injury recruits downstream targets like PLC $\gamma 1$, leading to KCC2 hypofunction. Here, the antiseizure efficacy of TrkB agonists LM22A-4, HIOC, and deoxygedunin (DC) on PB-refractory seizures and postischemic TrkB pathway activation was investigated in a mouse model (CD-1, P7) of refractory neonatal seizures. LM, a BDNF loop II mimetic, rescued PB-refractory seizures in a sexually dimorphic manner. Efficacy was associated with a substantial reduction in the postischemic phosphorylation of TrkB at Y816, a site known to mediate postischemic KCC2 hypofunction via PLC $\gamma 1$ activation. LM rescued ischemia-induced phospho-KCC2-S940 dephosphorylation, preserving its membrane stability. Full TrkB agonists HIOC and DC similarly rescued PB refractoriness. Chemogenetic inactivation of TrkB substantially reduced postischemic neonatal seizure burdens at P7. Sex differences identified in developmental expression profiles of TrkB and KCC2 may underlie the sexually dimorphic efficacy of LM. These results support a potentially novel role for the TrkB receptor in the emergence of age-dependent refractory neonatal seizures.

Authorship note: PAK and BJS contributed equally to this work.

Conflict of interest: SDK is listed as an author on US patent 10525024B2, "Methods for rescuing phenobarbitalresistance of seizures by ANA-12 or ANA-12 in combination with CLP290."

Copyright: (ㄷ) 2020, American Society for Clinical Investigation.

Submitted: January 6, 2020

Accepted: May 14, 2020

Published: June 18, 2020.

Reference information: /CI Insight. 2020;5(12):e136007.

https://doi.org/10.1172/jici.

insight.136007.

\section{Introduction}

Excitotoxic injury has been shown to phosphorylate tyrosine receptor kinase B (TrkB) pathway signaling (13). TrkB is activated by its endogenous ligand, the neurotrophin brain-derived neurotropic factor (BDNF), and leads to the activation of multiple intracellular signaling cascades, including 3 major downstream signaling cascades: phospholipase $\mathrm{C} \gamma 1$ (PLC $\gamma 1)$ /protein kinase $\mathrm{C}$, mitogen-activated protein kinase (MAP/ERK kinase), and the phosphatidylinositol 3-kinase/Akt (4). We have previously demonstrated that the postischemic activation of the TrkB/PLC $\gamma 1$ pathway results in the hypofunction of the $\mathrm{K}-\mathrm{Cl}$ cotransporter (KCC2) in a mouse model of acute neonatal ischemia with phenobarbital-refractory (PB-refractory) seizures (5).

The electroneutral cation-chloride transporter $\mathrm{KCC} 2$ is the primary neuronal $\mathrm{Cl}^{-}$extruder and enables hyperpolarizing GABAergic inhibition in the brain. The residue Ser940 (S940) on the C-terminus of KCC2 is associated with its membrane stability and chloride extrusion capacity $(6,7)$. The developmental switch in GABAergic signaling from depolarizing to hyperpolarizing (8) is enabled by an age-dependent increase of KCC2 expression (9). In the neonatal period, KCC2 expression is low and GABA is depolarizing (8-10). In addition, $\mathrm{KCC} 2$ is susceptible to degradation after excitotoxic injury $(2,11,12)$. In our characterized $\mathrm{CD}-1$ mouse model of ischemic neonatal seizures, KCC2 underwent degradation and dephosphorylation of S940 (5), rendering the antiseizure medication PB inefficacious (5) because PB is a positive allosteric modulator of $\mathrm{GABA}_{\mathrm{A}}$ receptors (13). Prevention of BDNF/TrkB-mediated KCC2 hypofunction rescued $\mathrm{PB}$ refractoriness in CD-1 pups (5). We hypothesized that the BDNF mimetic LM22A-4 (LM) (14) would interfere with the postischemic BDNF/TrkB signaling underlying the emergence of refractoriness.

This study used a characterized model of PB-refractory neonatal ischemic seizures at P7 and PB-responsive ischemic seizures at P10 $(2,5,15)$. The efficacy of the BDNF loop II mimetic LM 
$(0.25 \mathrm{mg} / \mathrm{kg}$ [LM0.25]) was compared with 2 graded doses $(0.025 \mathrm{mg} / \mathrm{kg}$ [LM0.025] and $2.5 \mathrm{mg} / \mathrm{kg}$ [LM2.5]). Additionally, LM was compared with the novel full TrkB agonists HIOC (16) and deoxygedunin (DG) (17). HIOC is an $\mathrm{N}$-acetylserotonin (NAS) derivative that exhibits more robust neurotrophic effects than NAS in a TrkB-dependent manner $(16,18)$. DG is a naturally occurring compound in the gedunin family that has shown robust neuroprotective properties in rats in a TrkB-dependent manner (17). The developmental profile of TrkB in neonatal brains is shown to decrease with age (19-22) and was also investigated here in the CD-1 strain. The role of the TrkB receptor in neonatal seizure susceptibility was investigated using a chemogenetic mouse model.

\section{Results}

$L M$ rescued neonatal PB-refractory seizures in a sex-dependent manner. Ischemia-induced seizures in P7 CD-1 pups are PB refractory (15). Previously, it has been shown that ANA12, a small-molecule TrkB antagonist, significantly rescued $\mathrm{PB}$ refractoriness by blocking BDNF/TrkB pathway activation in a dose-dependent manner $(2,5)$. To evaluate the efficacy of LM, a small-molecule TrkB partial agonist on rescuing PB refractoriness, P7 pups were either given LM immediately after ligation (post-LM group) or 2 hours before ligation (pre-LM group) per the experimental paradigm (Figure 1A). Continuous 2-hour video EEG/EMG recordings were used to identify and quantify postischemic electrographic seizure burdens (Figure 1, B-D). The seizure burden in the ligate $+\mathrm{PB}$ group remained unchanged after $\mathrm{PB}$ administration, indicating $\mathrm{PB}$ refractoriness. The post- and pre-LM groups both showed significant rescue of $\mathrm{PB}$ refractoriness (Figure 1, B-E). Clustering of ictal events was noted in the ligate $+\mathrm{PB}$ raster plot after $\mathrm{PB}$ injection (i.e., ligate $+\mathrm{PB}$ second hour vs. first hour) without overall reduction in seizure burdens (Figure 1D). Both the post- and pre-LM groups also showed similar clustering of ictal events associated with a concomitant significant reduction in overall seizure burdens after $\mathrm{PB}$ administration (Figure 1, D and E). When quantified as a percentage of seizure suppression over the first hour baseline, intervention with $\mathrm{LM}+\mathrm{PB}$ significantly increased seizure suppression in P7 pups (Figure 1E), in contrast to intervention with only PB, which failed to show seizure suppression $(-4.46 \% \pm 5.34 \%)$.

Neonatal seizure susceptibility and antiseizure medication efficacy have been shown to be sexually dimorphic $(5,15,23-25)$; therefore, sex as a biological variable was investigated. At P7, both sexes were PB refractory. Female pups were significantly responsive to LM intervention in the post- and pre-LM groups, whereas male pups only responded in the pre-LM group (Figure 1F). Furthermore, the percentage of seizure suppression in female pups was not significantly different between the post- and pre-LM groups (Figure $1 \mathrm{~F}$ ), demonstrating that preischemic LM intervention did not provide females any additional benefit in the rescue of $\mathrm{PB}$ refractoriness. However, female pups in the post-LM group showed a significantly greater percentage of seizure suppression than male pups in the post-LM group (Figure 1F), highlighting important sex differences in the efficacy of LM at P7.

As previously characterized for the model, neonatal ischemic seizures at P10 were PB responsive in both sexes (ref. 15 and Figure 1, G and H), supporting a developmental influence on PB efficacy. At P10, only the pre-LM group showed significant improvement in PB efficacy (Figure 1G). In contrast to P7, no sexual dimorphism was evident in the post- or pre-LM treatment groups at P10 (Figure 1H), highlighting the role of developmental age in sexual dimorphism. In summary, LM intervention significantly rescued $\mathrm{PB}$ refractoriness at $\mathrm{P} 7$ and improved $\mathrm{PB}$ efficacy at $\mathrm{P} 10$.

No dose-dependent effects for graded doses of LM. To evaluate the dose-dependent efficacy of LM, 2 additional graded doses of 10-fold lower and higher LM (0.025 vs. 0.25 vs. $2.5 \mathrm{mg} / \mathrm{kg})$ were evaluated for PB-refractory seizures at P7. The post-LM2.5 group significantly suppressed PB-refractory seizures by $42.77 \% \pm 9.98 \%$, similar to but not significantly better than the post-LM $0.25-\mathrm{mg} / \mathrm{kg}$ dose (2-tailed $t$ test, $P=0.6375$ ). In contrast to the post-LM0.25 and post-LM2.5 groups, the post-LM0.025 group had significantly aggravated first-hour seizure burden compared with ligate $+\mathrm{PB}$ (Supplemental Figure 1A; supplemental material available online with this article; https://doi.org/10.1172/jci. insight.136007DS1). The second-hour seizure burden remained similar to the ligate+PB group (Supplemental Figure 1B), signifying a lack of efficacy in rescuing refractoriness when administered acutely after ischemia. In contrast to the $0.25-\mathrm{mg} / \mathrm{kg}$ dose, there were no significant differences in seizure suppression between sexes in the post- and pre-LM2.5 groups. The aggravation in seizure burden in the post-LM0.025 group and significant seizure suppression in both the post-LM0.25 and post-LM2.5 groups indicated a nonlinear dose-response curve (26). 
A

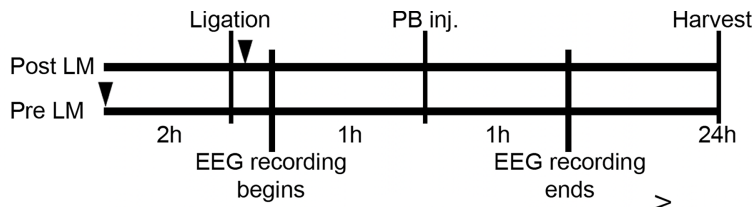

B P7 VEEG

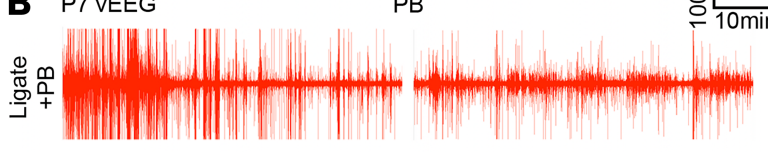

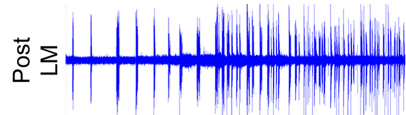

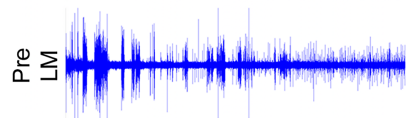

\section{C}
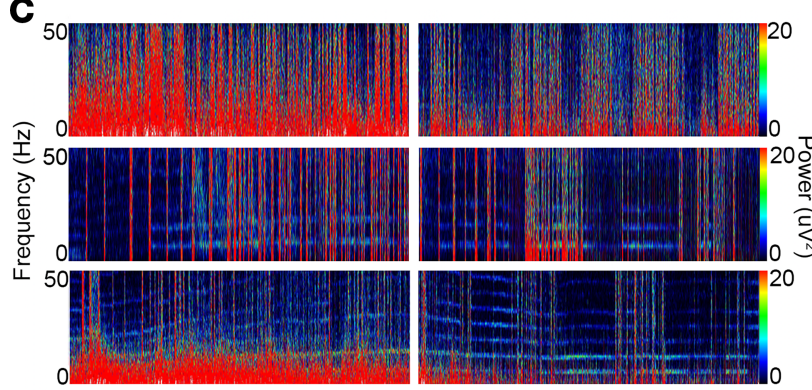

D

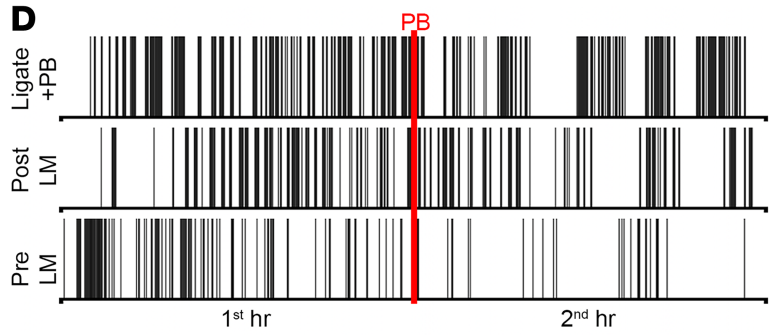

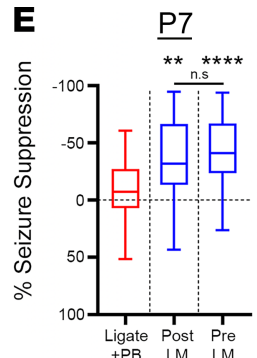
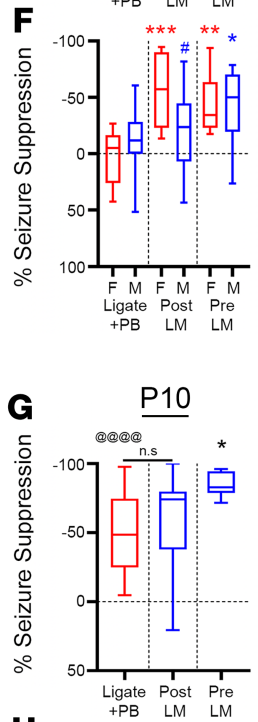

H

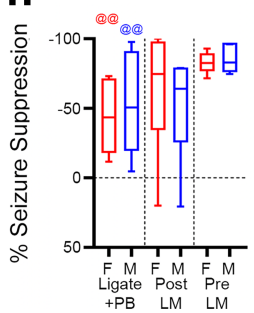

Figure 1. LM significantly rescued PB refractoriness. (A) Experimental paradigm to evaluate LM efficacy in a mouse model of ischemic neonatal seizures. Pups were randomly assigned to treatment groups (see Supplemental Table 1 for sample sizes). Black arrowheads indicate time point of LM intervention. (B) Representative EEG traces, (C) power spectrograms $(0-50 \mathrm{~Hz}$ ), and $(\mathbf{D})$ raster plots showing significantly rescued PB refractoriness in post- and pre-LM0.25 pups. Red line, time point of PB administration. (E) EEG percentage of seizure suppression for ligate+PB-, post-LM-, and pre-LM-treated P7 pups. ${ }^{* *} P<0.01$ (post-LM vs. ligate+PB), ${ }^{* * *} P<0.0001$ (pre-LM vs. ligate+PB) by 1-way ANOVA. Number of mice $n=28$ (ligate+PB), 26 (post-LM), 27 (pre-LM). (F) EEC percentage of seizure suppression by sex for ligate+PB-, post-LM-, and pre-LM-treated P7 pups. ${ }^{*} P<0.05$ (male pre-LM vs. male ligate+PB), ${ }^{* *} P<0.01$ (female pre-LM vs. female ligate+PB), ${ }^{* *} P<0.001$ (female post-LM vs. female ligate+PB) by 2 -way ANOVA. The \# indicates within-group sex differences; ${ }^{P} P<0.05$ by 2-tailed $t$ test. $n=16 / 12$ (ligate+PB [M/F]), 14/12 (post-LM), 14/13 (pre-LM). (G) EEC percentage of seizure suppression for ligate+PB-, post-LM-, and pre-LM-treated P10 pups. ${ }^{*} P<0.01$ (pre-LM vs. ligate+PB) by 1-way ANOVA. The @ indicates difference between P7 and P10; $@ @ @ @ P<0.0001$ by 2-tailed $t$ test. $n=$ 11 (ligate+PB), 13 (post-LM), 11 (pre-LM). (H) EEG percentage of seizure suppression by sex for ligate+PB-, post-LM-, and pre-LM-treated P10 pups. The @ indicates sex differences between P7 and P10; ${ }^{\circledR} P<0.01$ by 2-tailed $t$ test. $n=6 / 5$ (ligate+PB [M/F]), 6/7 (post-LM), 5/6 (pre-LM). Box-and-whisker plots show quartiles with median and minima and maxima at the bottom and top whiskers, respectively.

To evaluate the effect of TrkB agonists LM and HIOC on plasma membrane expression of TrkB and $\mathrm{KCC} 2$, naive $\mathrm{P} 7$ mouse pup brain slices were incubated with either $0.75-\mathrm{mM}$ - or 7.5-mM-graded doses of LM or a 1.7-mM dose of HIOC. These in vitro doses mimicked the in vivo doses used to study antiseizure efficacies $(0.25 \mathrm{mg} / \mathrm{kg}, 2.5 \mathrm{mg} / \mathrm{kg}$, and $5 \mathrm{mg} / \mathrm{kg}$, respectively). Incubation of naive P7 brain slices with the TrkB agonists showed no significant increase in phospho-TrkB-Y816 (p-TrkB-Y816) and KCC2 expression at the plasma membrane (Supplemental Figure 1, C and D). Similarly, the ratio of p-TrkB-Y816 to total TrkB at 
the membrane failed to show a significant dose-dependent increase with either LM or HIOC (Supplemental Figure 1E). The S940/KCC2 ratios at the membrane were also not significantly modulated by LM or HIOC (Supplemental Figure 1F). Plasma membrane proteins were normalized to caveolin (TrkB) or transferrin receptor-1 (TfR; KCC2). Both caveolin and TfR showed significantly higher fluorescence at the membrane (Supplemental Figure 1, G and H, 2-way ANOVA, $P<0.005$ for all treatment groups). The ratios of caveolin and TfR in the cytosol were 2 to 3 orders of magnitude lower than the membrane, which demonstrated efficacious separation of cytosol and plasma membrane. Ratios of cytosolic to membrane fluorescence of caveolin and TfR were not significantly different across treatment groups (Supplemental Figure 1, G and H). These in vitro findings indicated that the rescue of KCC2 expression by LM and HIOC was specific to the postischemic activation of the BDNF/TrkB pathway in seizing pups. In the absence of the ischemic injury, the TrkB agonists did not significantly modulate either TrkB or KCC2 insertion at the membrane.

Effect of LM on frequency and duration of neonatal ischemic seizures. To investigate the seizure semiology, seizure burden was evaluated as the total amount of time spent seizing on EEG. Baseline seizure burdens (i.e., represented by the seizures during the first hour of EEG recording) for each treatment group in this study were not significantly different from each other at P7 (Figure 2A) and P10 (Figure 2B). At P7, the post- and pre-LM groups demonstrated significant reductions in seizure burdens during the second hour after $\mathrm{PB}$ administration (Figure 2A). In contrast, the ligate $+\mathrm{PB}$ group failed to reduce seizure burdens in the second hour, demonstrating PB refractoriness. At P10 after ischemic insult, baseline seizure burdens were significantly lower than at P7 (Figure 2B vs. 2A), as previously characterized for the model $(2,5,15)$. Both the post- and pre-LM groups showed significant reduction in seizure burdens after $\mathrm{PB}$ administration, similar to the ligate $+\mathrm{PB}$ group.

Analysis of the number of ictal events (Figure 2, C and D) in all treatment groups revealed that the significant seizure suppression (Figure 1) with LM intervention was driven by significant reductions in the number of ictal events at both P7 and P10 (Figure 2, C and D). PB refractoriness in the ligate+PB group at P7 was driven by a significant increase in ictal durations (Figure 2E). In contrast, ictal durations during the second hour in the post- and pre-LM groups were not significantly different at both P7 and P10 (Figure 2, E and F). P7 female pups in both the post- and pre-LM groups had significantly lower second-hour seizure burdens than their respective first hour (Figure 2G). In contrast, only males in the pre-LM group had significantly lower second-hour seizure burdens when compared with their respective first hour at $\mathrm{P} 7$ (Figure $2 \mathrm{G}$ ).

At P10, the first-hour seizure burdens for male and female pups in the ligate+PB group had significantly lower first-hour seizure burdens than their respective P7 counterparts (Figure 2H). In contrast to P7, the P10 ligate + PB group demonstrated differences between sexes in that only males had significantly lower seizure burdens in the second hour than their respective first hour. The females in the post-LM group at P10 had significantly lower seizure burdens in the second hour than their respective first hour, similar to P7. In contrast, P10 males and females in the pre-LM group had significantly lower second-hour seizure burdens than their respective first hour, similar to pre-LM at P7. The sex-dependent differences in ictal events and durations at both ages were not significant (Figure 2, I-L).

EEG power was not predictive of acute antiseizure medication efficacy. EEG power has been used as a proxy to determine seizure burden on acute induced seizures $(27,28)$. EEG power suppression was examined to evaluate the efficacy of LM intervention (for example, 10-minute EEG seizure trace; see Supplemental Figure 2, A-D). The ligate+PB group showed a similar percentage of EEG power suppression to the LM-treated groups (Supplemental Figure 2E), which was driven by reductions in the second-hour EEG powers in all treatment groups. Therefore, EEG spectral power evaluated in a subset of LM-treated P7 pups failed to estimate accurate seizure burdens in the ligate+PB and LM-treated groups (Supplemental Figure 2F), indicating the unreliability of EEG power to detect accurate seizure burdens. Overall EEG power diminishes with the occurrence of repeated ischemic seizures (29). This phenomenon has also been reported for clinical EEGs (30). The correlation between percentage of EEG power suppression and percentage of seizure suppression showed that quantification of EEG power alone could not accurately measure seizure burdens (Supplemental Figure 2G), similar to previous reports $(5,23)$.

TrkB inactivation facilitates postischemic amelioration of seizure susceptibility at $P 7$. To investigate the role of postischemic TrkB pathway activation at $\mathrm{P} 7$ in vivo, TrkB activation was chemogenetically inhibited by

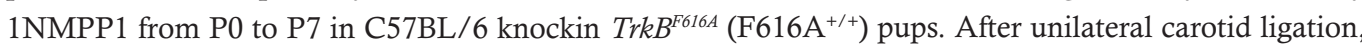
$\mathrm{WT}^{-/-}$pups administered 1NMPP1 showed significantly higher first-hour seizure burdens than $\mathrm{F}_{616 \mathrm{~A}^{+/+}}$ 
A

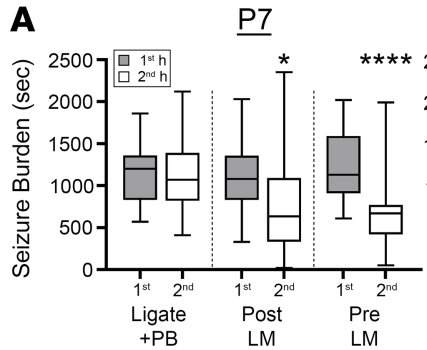

\section{B}

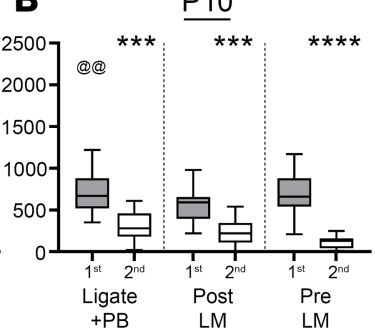

C

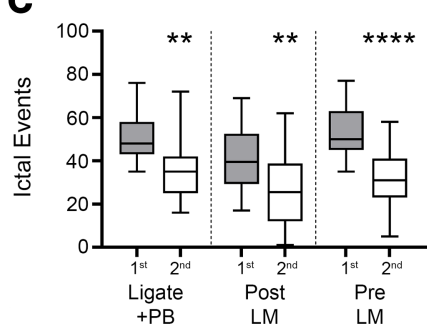

D
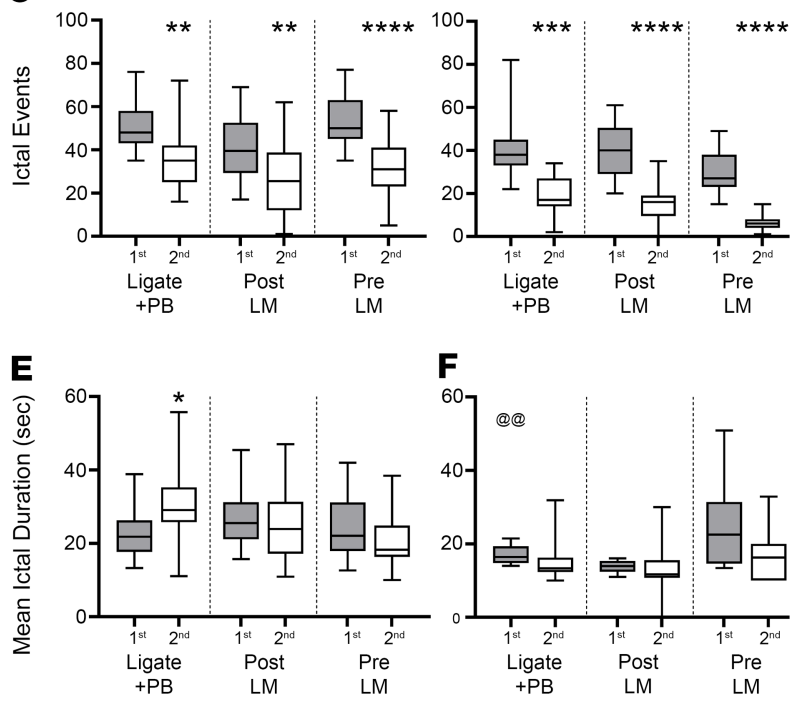
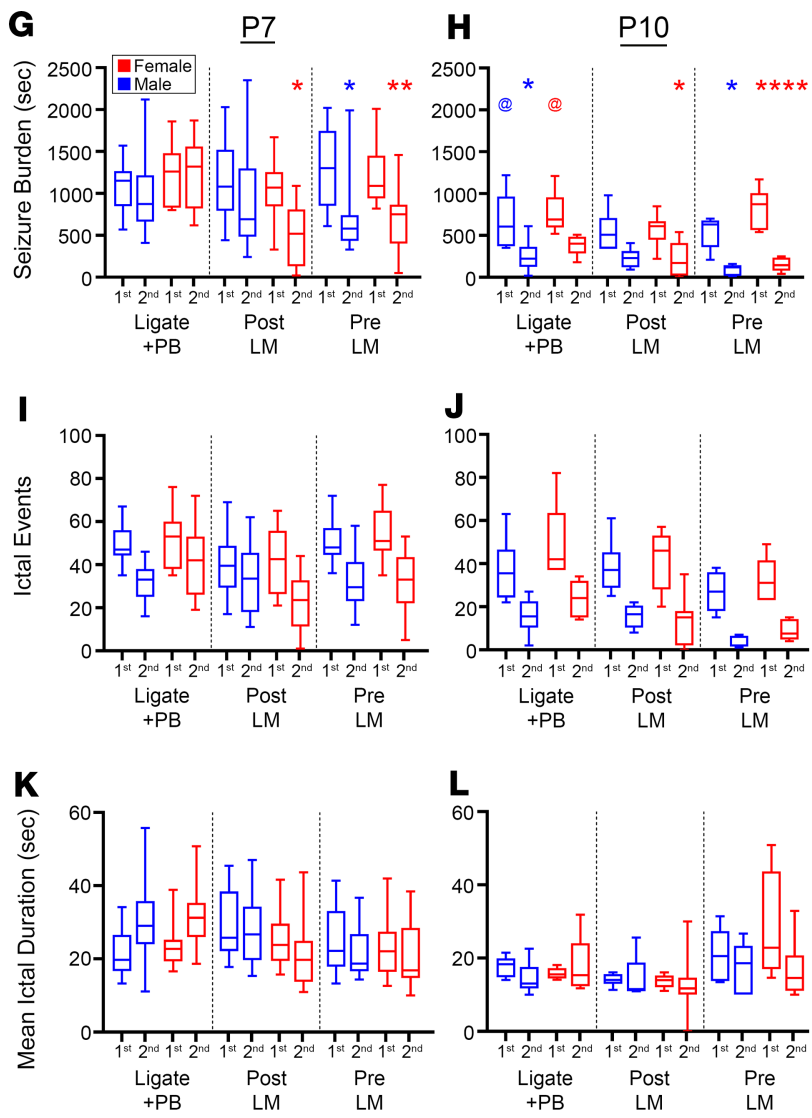

Figure 2. LM efficacy was sexually dimorphic and driven by significant reduction of ictal events, but not ictal durations, at both P7 and P10. (A) EEC seizure burdens for ligate+PB-, post-LM0.25-, and pre-LM0.25-treated P7 pups. ${ }^{*} P<0.05$ (second post-LM vs. first post-LM); ${ }^{* * *} P<0.0001$ (second pre-LM vs. first pre-LM) by 2-way ANOVA. (B) EEG seizure burdens for ligate+PB-, post-LM-, and pre-LM-treated P10 pups. ${ }^{* *} P<0.001$ (second ligate+PB vs. first ligate+PB, and second post-LM vs. first post-LM); ${ }^{* * *} P<0.0001$ (second pre-LM vs. first pre-LM) by 2-way ANOVA. The @ indicates differences between P7 and P10; ${ }^{@} P<0.01$ by 2 -tailed $t$ test. (C) EEG ictal events for ligate+PB-, post-LM-, and pre-LM-treated P7 pups. ${ }^{* *} P<0.001$ (second ligate+PB vs. first ligate+PB, and second post-LM vs. first post-LM); ${ }^{* * *} P<0.0001$ (second pre-LM vs. first pre-LM) by $2-$ way ANOVA. (D) EEG ictal events for ligate+PB-, post-LM-, and pre-LM-treated P10 pups. ${ }^{* *} P<0.001$ (second ligate+PB vs. first ligate+PB), ${ }^{* * *} P<0.0001$ (second post-LM vs. first post-LM, and second pre-LM vs. first pre-LM) by 2-way ANOVA. (E) EEG mean ictal durations for ligate+PB-, post-LM-, and pre-LM-treated P7 pups. ${ }^{*} P<0.05$ (second ligate+PB vs. first ligate+PB) by 2-way ANOVA. (F) EEG mean ictal durations for ligate+PB-, post-LM-, and pre-LM-treated P10 pups. @@ $P<0.01$ by 2 -tailed $t$ test. (G) EEG seizure burdens by sex for ligate+PB-, post-LM-, and pre-LM-treated P7 pups. ${ }^{*} P<0.05$ (second female post-LM vs. first female post-LM, and second male pre-LM vs. first male pre-LM); ${ }^{*} P<0.01$ (second female pre-LM vs. first female pre-LM) by $2-w a y ~ A N O V A$. (H) EEG seizure burdens by sex for ligate+PB-, post-LM-, and pre-LM-treated P10 pups. ${ }^{*} P<0.05$ (second male ligate+PB vs. first male ligate+PB, second female post-LM vs. first female post-LM, and second male pre-LM vs. first male pre-LM); ${ }^{* * *} P<0.0001$ (second female pre-LM vs. first female pre-LM) by 2 -way $A N O V A$; ${ }^{*} P<$ 0.05 by 2-tailed $t$ test. (I) EEG ictal events by sex for ligate+PB-, post-LM-, and pre-LM-treated P7 and (J) P10 pups. (K) EEG mean ictal durations by sex for ligate+PB-, post-LM-, and pre-LM-treated P7 and (L) P10 pups. Number of P7 mice: $n=28$ [16/12] (ligate+PB [M/F]), 26 [14/12] (post-LM), 27 [14/13] (preLM). Number of P10 mice: $n=11$ [6/5] (ligate+PB), 13[6/7] (post-LM), 11 [5/6] (pre-LM). Box-and-whisker plots show quartiles with median with minima and maxima at the bottom and top whiskers, respectively.

pups administered 1NMPP1 (Figure 3, A-D), demonstrating that the chemogenetic inactivation of TrkB significantly reduced the postischemic seizure susceptibility in P7 neonatal pups. EEG seizure burdens were significantly lower in the $\mathrm{F}_{6} 16 \mathrm{~A}^{+/+}$pups in the first hour after ischemia. EEG seizure burdens evaluated by sex found no sex-dependent differences. PB administration at the end of 1 hour in $\mathrm{WT}^{-/-}$C57BL/6 pups (Figure 3D) was efficacious. These results support the established importance of genetic background on ischemia and seizures in that phenotypic severity is strain dependent (31-33). The CD-1 strain showed the phenotype severity and emergence of refractory neonatal seizures with added translational value in comparison with the C57BL/6 strain. Overall, these results demonstrated that postischemic activation of the BDNF/TrkB signaling cascade plays a crucial role in neonatal seizure susceptibility.

Postischemic TrkB/PLC 1 pathway activation at $P 7$ was rescued by $L M$ intervention. Ischemic insults are known to induce BDNF/TrkB pathway activation $(5,34)$, and phosphorylation of Y816 on TrkB activates the PLC $\gamma 1$ pathway, which has been implicated both acutely (5) and chronically in epileptogenesis (35). 

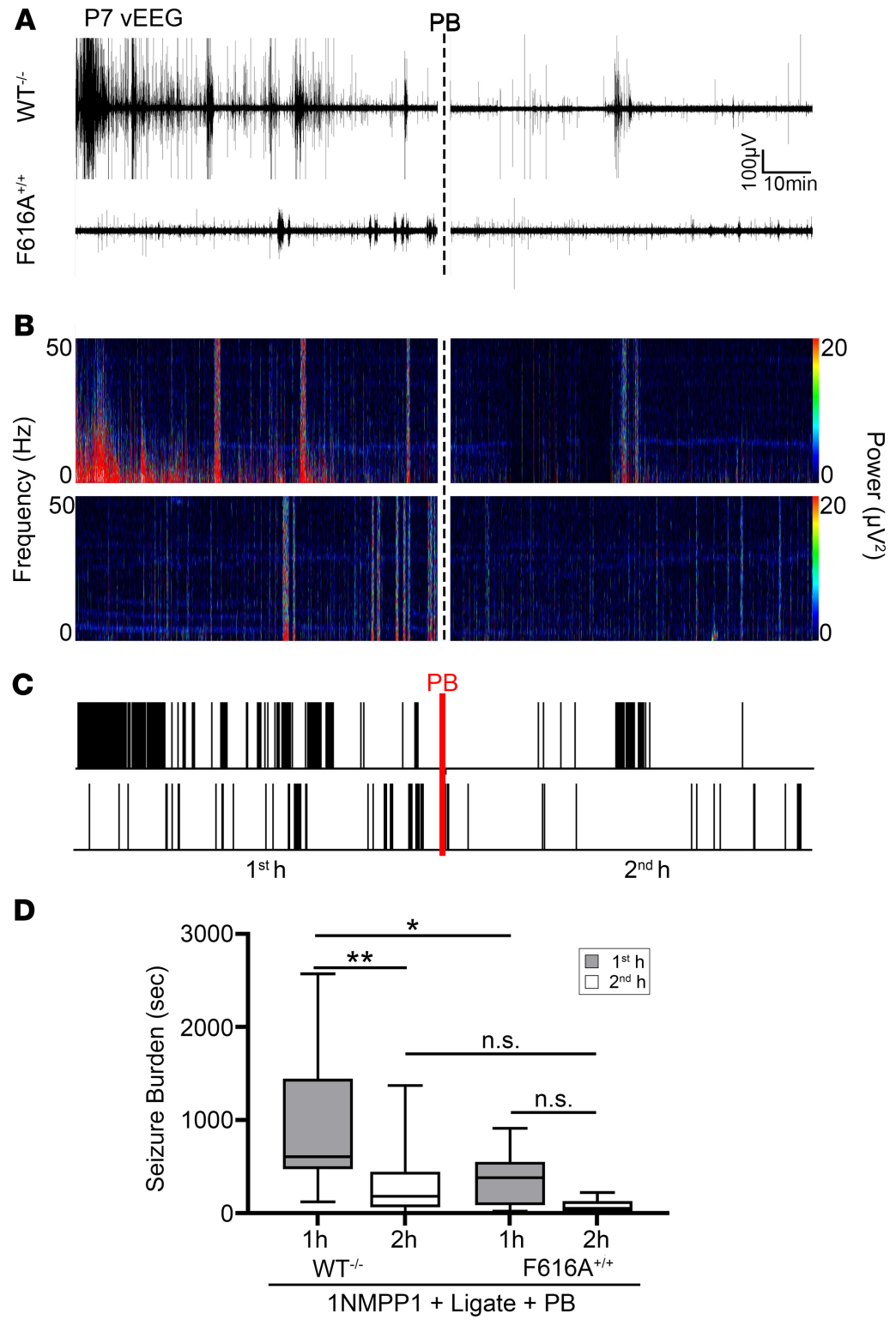

Figure 3. Postischemic TrkB activation underlies neonatal seizure susceptibility. (A) Representative EEC traces in $\mathrm{WT}^{-1-}$ and $\mathrm{F} 616 \mathrm{~A}^{+/+}$pups, (B) power spectrograms $(0.5-50 \mathrm{~Hz})$, and (C) raster plots showed significantly lower first-hour postischemic EEG seizure burdens in $\mathrm{F} 616 \mathrm{~A}^{+/+}$. Dotted black and red lines indicate time point of PB administration. (D)

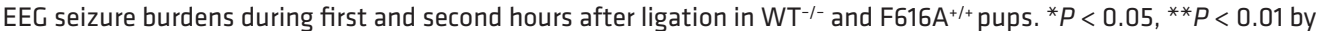

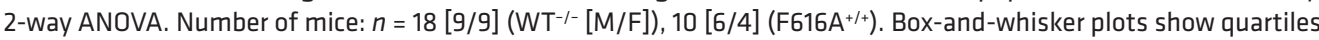
with median with minima and maxima at the bottom and top whiskers, respectively.

Pups in the ligate+PB group showed a significant increase of TrkB and p-TrkB-Y816 expression ipsilateral and contralateral to ischemic insult 24 hours after ischemia (Figure 4, A-C), indicating global TrkB activation in the unilateral model. With the unilateral ischemia model used in this study, ipsilateral versus contralateral hemispheric differences in protein expression were analyzed. The post-LM group showed attenuated TrkB expression ipsilateral to ischemic insult (Figure 4A). The p-TrkB-Y816/TrkB ratio of the ligate $+\mathrm{PB}$ group was significantly lower ipsilateral to ischemic insult (Supplemental Figure 3A). The post- and pre-LM groups both significantly rescued postischemic TrkB pathway activation. The ratios of $\mathrm{p}-$ TrkB-Y816 to total TrkB showed no significant differences between treatment groups (Supplemental Figure 3A). Postischemic TrkB activation was analyzed by sex, and no significant sex-dependent differences were found. Graded doses of LM (LM0.025 and LM2.5) did not significantly affect the ratio of p-TrkB-Y816 to total TrkB (Supplemental Figure 3, E and F). 
Total PLC $\gamma 1$ expression was not significantly modulated by ischemic insult or post- and pre-LM intervention (Figure 4, D and E). In contrast, phospho-PLC $\gamma 1-Y 783$ (p-PLC $\gamma 1-Y 783$ ) expression was significantly higher both ipsilateral and contralateral to ischemic insult, which was rescued in the post-LM and pre-LM groups (Figure 4, D and F). PLC $\gamma 1$ and p-PLC $\gamma 1-Y 783$ expression levels were also significantly lower ipsilateral to ischemic insult (Figure 4, D-F). The ratio of p-PLC $\gamma 1-Y 783$ to total PLC $\gamma 1$ was elevated in the ligate $+\mathrm{PB}$ group, which was rescued in the post- and pre-LM treatment groups and demonstrated rescue of the pathological activation of the TrkB/PLC $\gamma 1$ pathway (Supplemental Figure 3B).

TrkB pathway activation is also known to activate downstream ERK1/2 signaling (36). At P7, pre-LM was the most efficacious treatment paradigm in reducing EEG seizure burdens when quantified as a percentage of seizure suppression (Figure $1 \mathrm{E}$ ) for both sexes. Therefore, ERK1/2 activation was investigated in the pre-LM group. The TrkB/ERK1/2 pathway was not significantly activated by ischemic insult and was not influenced by LM intervention. However, ERK1/2 expression was significantly lower ipsilateral to ischemic insult in the ligate+PB group (Figure 4, G and H). Furthermore, the pre-LM group showed significant activation of phospho-ERK1/2-T202/Y204 (p-ERK1/2-T202/Y204) (Figure 4I). These data indicate that LM differentially activated the TrkB/ERK1/2 pathway while simultaneously blocking ischemia-induced activation of the TrkB/PLC $\gamma 1$ pathway, which suggests that the TrkB/PLC $\gamma 1$ pathway activation may be BDNF loop II specific. The ratio of p-ERK1/2-T202/Y204 to total ERK1/2 was significantly higher in the pre-LM group both ipsilateral and contralateral to ischemic insult (Supplemental Figure 3C).

LM rescued postischemic KCC2 and p-KCC2-S940 degradation at P7. Postischemic TrkB/PLC $\gamma 1$ pathway activation and seizures led to ipsilateral KCC2 degradation in a model of unilateral ischemic insult $(2,5)$. The effect of TrkB/PLC $\gamma 1$ pathway activation on KCC2 expression was evaluated 24 hours after ischemia in the LM intervention groups. KCC2 and p-KCC2-S940 antibodies were validated using liver samples obtained from P7 pups (Figure $4 \mathrm{~K}$ ). The post- and pre-LM treatment groups significantly rescued the ipsilateral KCC2 degradation seen in the ligate+PB group (Figure 4, J-L). Phosphorylated KCC2-S940 is associated with KCC2 stability on the plasma membrane and thus the functionality of $\mathrm{KCC} 2$ as a $\mathrm{Cl}^{-}$extruder. Similar to KCC2, the ipsilateral p-KCC2-S940 dephosphorylation in the ligate $+\mathrm{PB}$ group was significantly rescued in the post- and pre-LM groups (Figure $4 \mathrm{M}$ ). The ratio of p-KCC2-S940 to total KCC2 was lower in the ligate+PB group and was rescued to naive levels in the post- and pre-LM0.25 groups (Supplemental Figure 3D). The low and high doses of LM (LM0.025 and LM2.5 groups, respectively) increased the ratio of p-KCC2-S940 to total KCC2 compared with the ligate $+\mathrm{PB}$ group. In summary, LM intervention rescued postischemic BDNF/TrkB/PLC $\gamma 1$ pathway activation, thus preventing KCC2 endocytosis and subsequent hypofunction.

Postischemic TrkB/PLC $\gamma 1$ pathway activation was not evident in ischemic P10 pups. In contrast to P7 pups, no increase in $\mathrm{p}-\mathrm{TrkB}-\mathrm{Y} 816$ expression was detected in any treatment group at P10 (Supplemental Figure $4, \mathrm{~A}-\mathrm{C}$ ). The pre-LM group showed significantly higher ratios of $\mathrm{p}-\mathrm{TrkB}-\mathrm{Y} 816$ to TrkB ipsilateral and contralateral to ischemic insult (Supplemental Figure 4D). PLC $\gamma 1$ expression was not different between treated and untreated pups, although post- and pre-LM pups showed ipsilateral downregulation of p-PLC $\gamma 1-Y 783$ (Supplemental Figure 4, E-G). Ratios of p-PLC $\gamma 1-Y 783$ to total PLC $\gamma 1$ were not affected by LM intervention (Supplemental Figure 4H). No significant KCC2 degradation was detected in the ligate $+\mathrm{PB}$ group (Supplemental Figure 4, I and J) after ischemia, although post-LM pups showed lower ipsilateral p-KCC2-S940 expression (Supplemental Figure 4, K and L). In summary, LM intervention showed mild activation of the TrkB pathway, but KCC2 levels were not significantly modulated at $\mathrm{P} 10$ when ischemic seizures were responsive to $\mathrm{PB}$.

TrkB agonists HIOC and DG rescued refractory ischemic seizures at P7 similarly to LM. The efficacy of 2 full TrkB agonists, HIOC (16) and DG (17), was investigated to determine whether the antiseizure efficacy of LM was TrkB site specific. At P7, HIOC demonstrated significant seizure suppression in both the post- and pre-HIOC groups (Figure 5A), indicating that LM antiseizure efficacy was not loop II site specific. Male pups in the pre-HIOC group demonstrated significant seizure suppression compared with male ligate $+\mathrm{PB}$ pups, in contrast to female pups (Figure 5B). No significant sex differences were noted in the post-HIOC group. The second-hour seizure burden in the pre-HIOC group was significantly lower than its first-hour baseline (Figure $5 \mathrm{C}$ ), in contrast to the post-HIOC and ligate $+\mathrm{PB}$ groups.

To evaluate the role of HIOC effects on TrkB pathway activation, expression levels of TrkB, ERK1/2, and KCC2 were examined 24 hours after ligation. Similar to LM intervention, the post- and pre-HIOC groups significantly bilaterally rescued TrkB pathway activation (Figure 5D) and p-TrkB-Y816 activation (Figure 5E). 
A

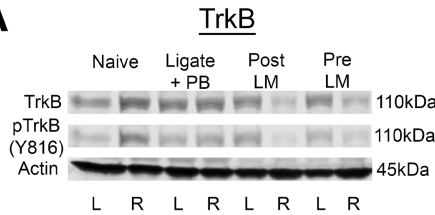

B
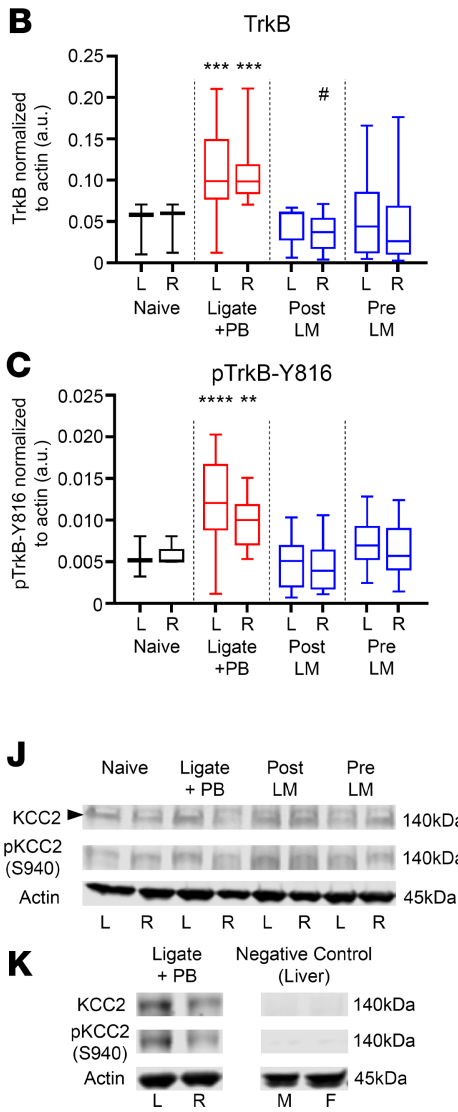

D

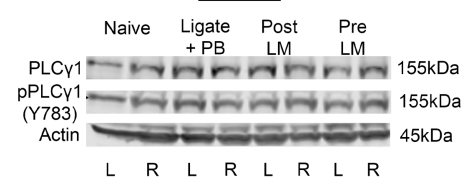

E
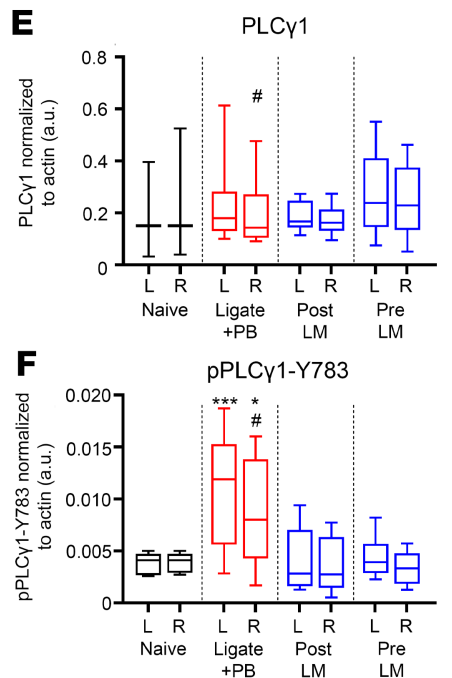

$\mathbf{L}$

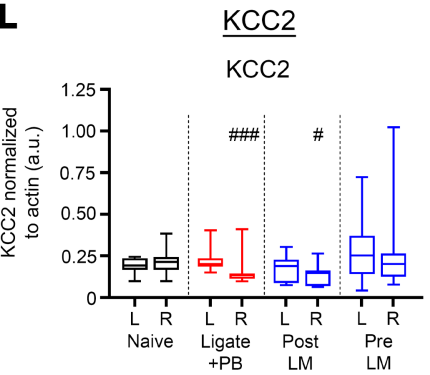

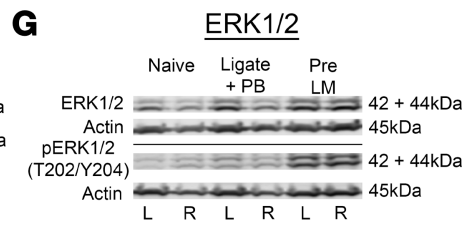
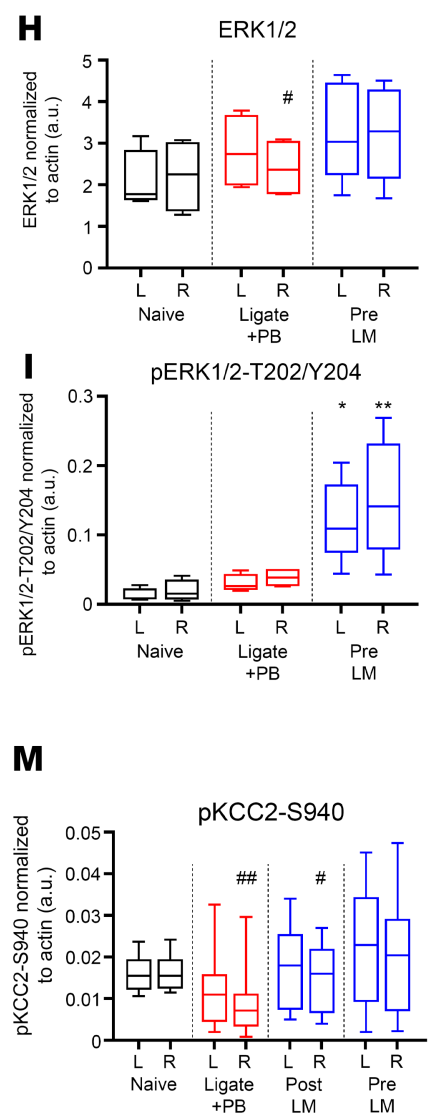

Figure 4. LM rescued postischemic TrkB/PLC $\gamma 1$ pathway activation, activated the TrkB/ERK1/2 pathway, and rescued ipsilateral KCC2 degradation. All proteins of interest were normalized to housekeeping protein $\beta$-actin. Phosphoproteins were also normalized to their respective total protein (see Supplemental Figure 3). The * indicates differences between treatment group and naive controls; the \# indicates differences between contralateral and ipsilateral hemispheres within groups. (A) Representative Western blots showing TrkB and p-TrkB-Y816 expression for all treatment groups. (B) Contralateral $(\mathrm{L})$ and ipsilateral $(\mathrm{R})$ TrkB expression 24 hours after ischemic insult. ${ }^{* *} P<0.001$ by 1 -way ANOVA. ${ }^{*} P<0.05$ by 2 -tailed $t$ test. (C) Contralateral ( $\mathrm{L}$ ) and ipsilateral (R) $p$-TrkB-Y816 expression 24 hours after ischemic insult. ${ }^{* *} P<0.01,{ }^{* * *} P<0.0001$ by 1-way ANOVA. (D) Representative Western blots showing PLC $\gamma 1$ and $p-P L C \gamma 1-Y 783$ expression. (E) Contralateral (L) and ipsilateral (R) PLC $\gamma 1$ expression 24 hours after ischemic insult. ${ }^{\#} P<0.05$ by 2 -tailed $t$ test. $(\mathbf{F})$ Contralateral $(\mathrm{L})$ and ipsilateral $(\mathrm{R}) \mathrm{p}-\mathrm{PLC} \gamma 1-\mathrm{Y} 783$ expression 24 hours after ischemic insult. ${ }^{*} P<0.05,{ }^{* * *} P<0.001$ by 1 -way ANOVA. ${ }^{*} P<0.05$ by 2-tailed $t$ test. (G) Representative Western blots showing ERK1/2 and p-ERK1/2-T202/Y204 expression. (H) Contralateral (L) and ipsilateral (R) ERK1/2 expression 24 hours after ischemic insult. ${ }^{\#} P<0.05$ by 2-tailed $t$ test. (I) Contralateral (L) and ipsilateral (R) $p$-ERK1/2-T202/Y204 expression 24 hours after ischemic insult. ${ }^{*} P<0.05,{ }^{* *} P<0.01$ by 1-way ANOVA. (J) Representative Western blots showing KCC2 and $p-K C C 2-S 940$ expression. (K) Representative Western blot showing lack of KCC2 and p-KCC2-S940 band in liver samples from P7 pups. (L) Contralateral (L) and ipsilateral (R) KCC2 expression 24 hours after ischemic insult. ${ }^{P} P<0.05$, $\# \# P<0.001$ by 2-tailed $t$ test. (M) Contralateral (L) and ipsilateral (R) $p$-KCC2-S940 expression 24 hours after ischemic insult. ${ }^{\#} P<0.05,{ }^{\#} P<0.01$ by 2 -tailed $t$ test. Number of mice: $n=13$ [6/7] (naive [M/F]), 19 [10/9] (ligate+PB), 13 [5/8] (post-LM), 22 [11/11] (pre-LM). Box-and-whisker plots show quartiles with median with minima and maxima at the bottom and top whiskers, respectively.

Total ERK1/2 expression was not significantly affected by ischemic insult or any HIOC treatment (Figure $5 \mathrm{~F}$ ), similar to LM intervention (Figure $4 \mathrm{H}$ ). In contrast to pre-LM intervention (Figure 4I), the post-HIOC and pre-HIOC groups did not demonstrate an increase in p-ERK1/2-T202/Y204 expression (Figure 5G). In summary, the differences in LM versus HIOC-mediated downstream ERK1/2 phosphorylation may depend upon site-specific TrkB binding.

$\mathrm{KCC} 2$ expression decreased unilaterally in the ipsilateral ischemic hemisphere in the ligate $+\mathrm{PB}$ group (Figure $5 \mathrm{H}$ ). The post-HIOC and pre-HIOC groups demonstrated significant rescue of KCC2 and pKCC2-S940 (Figure 5I) expression in the ipsilateral hemisphere. In summary, HIOC and LM had similar 


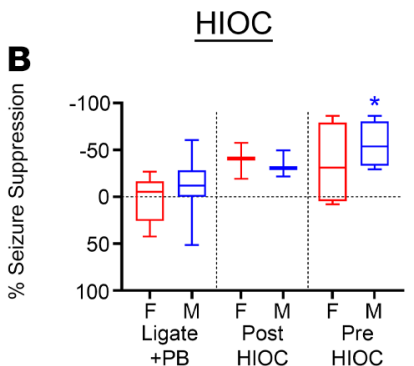

D

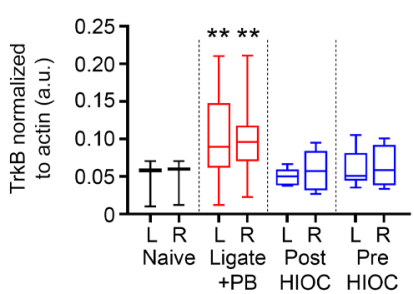

E
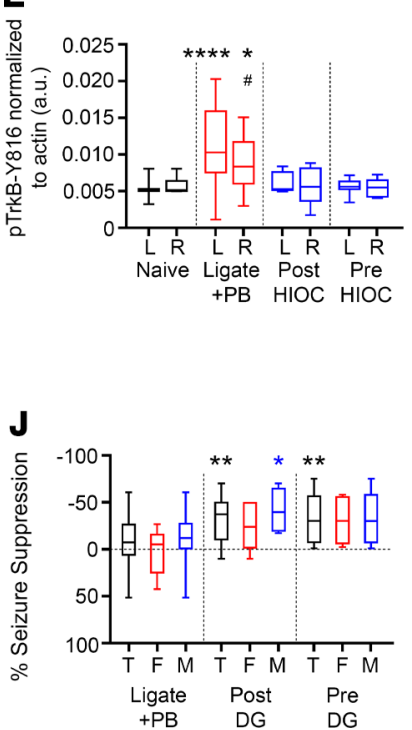

$\mathbf{F}$

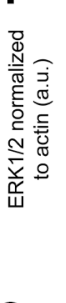

\section{G}
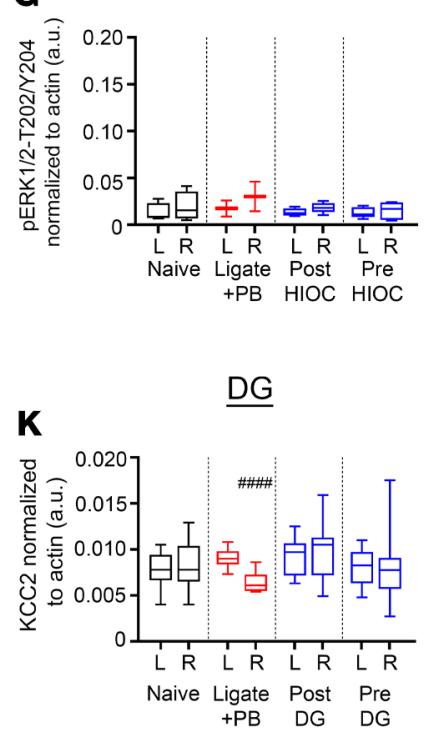

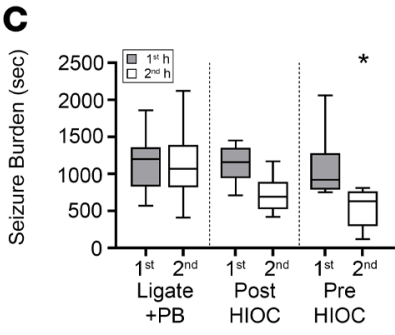

H
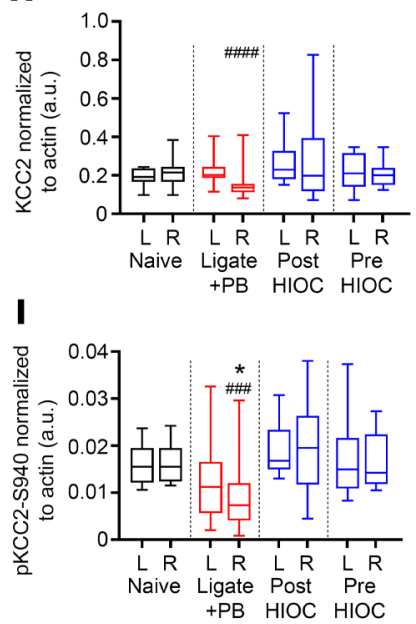

Figure 5. HIOC and DC significantly rescued PB-refractory seizures at P7. (A) EEC percentage of seizure suppression for ligate+PB-, post-HIOC-, and pre-HIOC-treated P7 pups. ${ }^{*} P<0.05$, ${ }^{* *} P<0.01$ by 1 -way ANOVA. Number of mice (EEC): $n=28$ [16/12] (ligate+PB [M/F]), 6 [3/3] (post-HIOC), 8 [4/4] (pre-HIOC). (B) EEG percentage of seizure suppression by sex for ligate+PB-, post-HIOC-, and pre-HIOC-treated P7 pups. ${ }^{*} P<0.05$ by 2 -way ANOVA. (C) EEC seizure burdens for ligate+PB-, post-HIOC-, and pre-HIOC-treated P7 pups. ${ }^{*} P<0.05$ by 2 -way ANOVA. (D) Contralateral $(\mathrm{L})$ and ipsilateral $(\mathrm{R})$ TrkB expression 24 hours after ischemic insult for all treatment groups. ${ }^{*} P<$ 0.01 by 1 -way ANOVA. All proteins were normalized to housekeeping protein $\beta$-actin. Number of mice (Western blot): $n=13$ [6/7] (naive), 19 [10/9] (ligate+PB), 5 [3/2] (post-HIOC), 6 [4/2] (pre-HIOC). (E) Contralateral (L) and ipsilateral $(R) p-T r k B-Y 816$ expression 24 hours after ischemic insult. ${ }^{*} P<0.05$. ${ }^{* * *} P<0.0001$ by 1 -way ANOVA. The \# indicates differences between contralateral and ipsilateral hemispheres within groups; ${ }^{P} P<0.05$ by 2 -tailed $t$ test. (F) Contralateral (L) and ipsilateral (R) ERK1/2 expression 24 hours after ischemic insult. (C) Contralateral (L) and ipsilateral (R) p-ERK1/2-T202/Y204 expression 24 hours after ischemic insult. (H) Contralateral (L) and ipsilateral (R) KCC2 expression 24 hours after ischemic insult. \#\#\# $P<0.0001$ by 2 -tailed $t$ test. (I) Contralateral (L) and ipsilateral (R) p-KCC2-S940 expression 24 hours after ischemic insult. ${ }^{*} P<0.05$ by 1 -way ANOVA. ${ }^{\# \# ~} P<0.001$ by 2 -tailed $t$ test. (J) EEC percentage of seizure suppression for ligate+PB, post-DG, and pre-DC treatment groups plotted as total data and split by sex. (T, total). ${ }^{*} P<0.05,{ }^{* *} P<0.01$ by 2 -way ANOVA. Horizontal dotted line represents $0 \%$ suppression. Number of mice (EEC): $n=28$ [16/12] (ligate+PB), 16 [8/8] (post-DG), 15 [10/5] (pre-DC). (K) Contralateral $(\mathrm{L})$ and ipsilateral $(\mathrm{R}) \mathrm{KCC} 2$ expression 24 hours after ischemic insult. The \# signifies hemispheric differences within treatment groups. $\# \# \# P<0.0001$ by 2-tailed $t$ test. Number of mice (Western blot): $n=13$ [6/7] (naive), 19 [10/9] (ligate+PB), 6 [4/2] (post-DC), 7 [4/3] (pre-DG). (L) Contralateral (L) and ipsilateral (R) TrkB expression 24 hours after ischemic insult. ${ }^{* * *} P<0.0001$ by 1 -way ANOVA. Box-and-whisker plots show quartiles with median with minima and maxima at the bottom and top whiskers, respectively. 
effects on TrkB, p-TrkB-Y816, KCC2, p-KCC2-S940, and ERK1/2 expression. In contrast to LM, HIOC treatment did not result in activation of the p-ERK1/2-T202/Y204 downstream pathway.

Treatment of $\mathrm{P} 7$ ischemic pups with another full TrkB agonist, DG, also significantly rescued $\mathrm{PB}$ refractoriness in the post-DG and pre-DG groups (Figure 5J). Male pups in the post-DG group had significantly greater seizure suppression than male pups in the ligate+PB group (Figure 5J), in contrast to LM and HIOC treatments. No significant sex differences in seizure suppression were noted for full TrkB agonists HIOC and DG. Comparing both of these TrkB agonists with LM highlighted that male and female pups differentially responded to BDNF loop II mimetics and full TrkB agonists. However, the postischemic KCC2 degradation and TrkB pathway activation was rescued in the post- and pre-DG groups (Figure $5, \mathrm{~K}$ and $\mathrm{L}$ ), similar to interventions with LM and HIOC. The differential activation of the TrkB/ERK1/2 pathway downstream of BDNF/TrkB signaling elicited by the BDNF mimetic LM was not detected with the TrkB agonists HIOC and DG.

Differences of developmental profiles of TrkB and PLC 1 expression in naive CD-1 pups by sex. At P5, females expressed significantly higher levels of TrkB in the cortex compared with males (Figure 6, A and B). Developmentally, TrkB expression in the cortex of female pups declined significantly from P5 to P21. In contrast, TrkB expression in males did not decline significantly from P5 to P21. Female and male pups both showed significant decline of $\mathrm{p}$-TrkB-Y816 expression from P5 to P21 (Figure 6C). In the cortex, ratios of $\mathrm{p}$ TrkB-Y816 to total TrkB were not significantly different by sex or age (Figure 6D).

Similar to the results found in the cortex, TrkB expression in the hippocampi of female pups declined significantly from P5 to P21, whereas TrkB expression in males did not decline significantly (Figure 6, E and F). p-TrkB-Y816 expression also declined significantly in the hippocampi of female pups from P5 to P21 (Figure 6G). Ratios of p-TrkB-Y816 to total TrkB were not sexually dimorphic and did not change significantly between the ages of P5 and P21 in the hippocampus (Figure $6 \mathrm{H}$ ). In deep gray matter, TrkB expression decreased significantly between P5 and P21 in females, whereas TrkB expression remained stable and did not decline significantly in males (Figure 6, I and J). In both females and males from P5 to P21, pTrkB-Y816 expression decreased significantly (Figure 6, I and K). However, ratios of p-TrkB-Y816 to total TrkB were not sexually dimorphic and did not change significantly between the ages of P5 and P21 in the deep gray matter (Figure 6L). These results suggest that sexually dimorphic expression levels of TrkB in the cortex may underlie the sexually dimorphic seizure susceptibility and rescue of PB refractoriness with LM (Figure 1 and Figure 2) because neonatal seizures are cortical. Furthermore, the attenuation of p-TrkB-Y816 from P5 to P21 in both males and females suggests that TrkB pathway activation decreases with age.

At P5, females showed significantly higher PLC $\gamma 1$ and p-PLC $\gamma 1-Y 783$ expression in the cortex than at P21 (Supplemental Figure 5, A-C). In contrast, PLC $\gamma 1$ and p-PLC $\gamma 1-Y 783$ expression in males was not significantly different between age groups. The hippocampus and deep gray matter did not show significant differences between sexes in PLC $\gamma 1$ or p-PLC $\gamma 1-Y 783$ expression during development (Supplemental Figure 5, E-K). Similarly, ratios of p-PLC $\gamma 1-Y 783$ to total PLC $\gamma 1$ were not significantly different between sexes during development for all examined brain regions (Supplemental Figure 5, D, H, and L).

Differences in developmental expression profile of KCC2 in naive CD-1 pups by sex. Males showed a significant increase in KCC2 expression between P5 and P21, and P7 and P21, whereas females did not (Supplemental Figure 5, M and N). p-KCC2-S940 was not significantly dephosphorylated in either sex (Supplemental Figure $5, \mathrm{M}$ and $\mathrm{O}$ ). In contrast, only females showed significantly lower ratios of p-KCC2-S940 to total KCC2 in the cortex (Supplemental Figure 5P). In the hippocampus, males and females did not show a significant change in KCC2 expression (Supplemental Figure 5, Q and R). Between ages, p-KCC2-S940 expression and ratios of p-KCC2-S940 to total KCC2 were not found to be sexually dimorphic (Supplemental Figure 5, S and T). In deep gray matter, females and males both showed significantly lower expression of p-KCC2-S940 at P21 (Supplemental Figure 5, U-X). In summary, the developmental age-dependent decline in KCC2 expression demonstrated an opposite trend to TrkB expression during the same developmental period.

\section{Discussion}

The main findings of this study are the following: (a) Acute administration of LM, a small-molecule BDNF loop II mimetic, significantly rescued PB refractoriness in a mouse model of neonatal seizures. (b) LM was more efficacious in female pups at P7. (c) LM prevented ischemia-induced TrkB/PLC $\gamma 1$ pathway activation and subsequent KCC2 degradation while significantly increasing ERK1/2 phosphorylation. (d) The full TrkB agonists HIOC and DG also rescued PB refractoriness and ischemia-induced KCC2 degradation, indicating that the efficacy of LM was not site dependent. HIOC also 


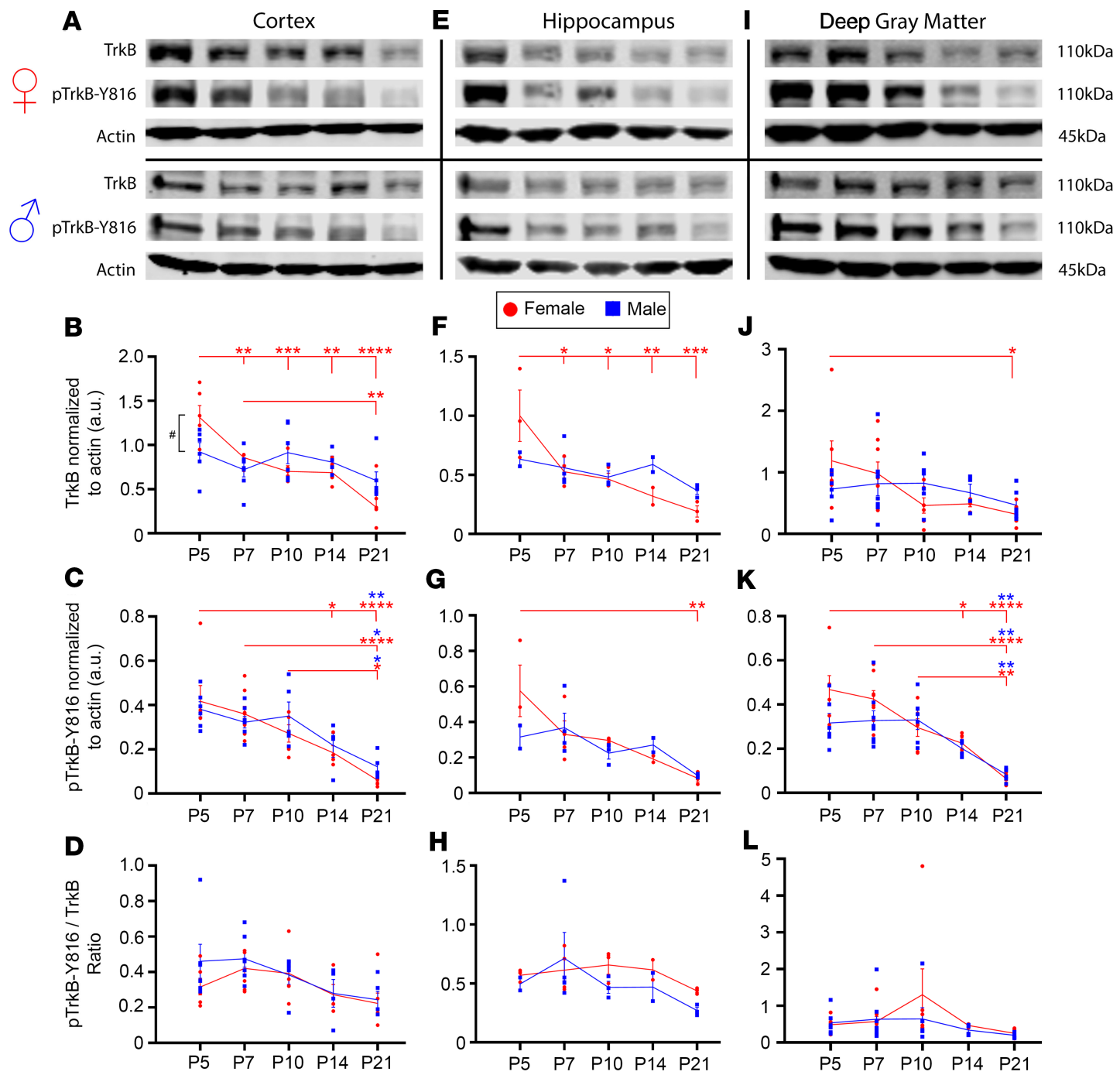

Figure 6. TrkB expression significantly decreased from $\mathbf{P 5}$ to $\mathbf{P 2 1}$ in a region-specific manner in naive female pups. All proteins were normalized to $\beta$-actin. (A) Representative Western blots showing TrkB and p-TrkB-Y816 expression in cortex for all female and male age groups. (B) TrkB expression in cortical tissue from P5 to P21. ${ }^{*} P<0.05$ signifies difference between sexes at a given age. ${ }^{* *} P<0.01,{ }^{* * *} P<0.001,{ }^{* * * *} P<0.0001$ by 2 -way ANOVA. (C) $p$-TrkB-Y816 expression in cortical tissue from P5 to P21. ${ }^{*} P<0.05,{ }^{* *} P<0.01,{ }^{* * * *} P<0.0001$ by 2-way ANOVA. (D) $p-T r k B-Y 816$ normalized to total TrkB in cortical tissue from P5 to P21. (E) Representative Western blots showing TrkB and p-TrkB-Y816 expression in hippocampus for all female and male age groups. (F) TrkB expression in hippocampal tissue from P5 to P21. ${ }^{*} P<0.05,{ }^{*} P<0.01,{ }^{* *} P<0.001$ by 2 -way ANOVA. (C) $p$ TrkB-Y816 expression in hippocampal tissue from P5 to P21. ${ }^{* *} P<0.01$ by 2-way ANOVA. (H) $p$-TrkB-Y816 normalized to total TrkB in cortical tissue from P5 to P21. (I) Representative Western blots showing TrkB and p-TrkB-Y816 expression in deep gray matter for all female and male age groups. (J) TrkB expression in deep gray matter from P5 to P21. ${ }^{*} P<0.05$ by 2-way ANOVA. (K) $p$-TrkB-Y816 expression in deep gray matter from P5 to P21. ${ }^{*} P<$ $0.05,{ }^{* *} P<0.01,{ }^{* * * *} P<0.0001$ by 2 -way ANOVA. (L) $p-T r k B-Y 816$ normalized to total TrkB in deep gray matter from P5 to P21. Number of mice per age group and region: $n \geq 4$ [2/2] (cortex [M/F]), $\geq 3$ [1/2] (hippocampus), $\geq 4$ [2/2] (deep gray matter). Dot plots show all data and the mean \pm 1 SEM. Red (female) and blue (male) lines connect the means across age groups.

prevented TrkB/PLC $\gamma 1$ pathway activation and KCC2 degradation but without increasing ERK1/2 phosphorylation. (e) At P10, seizures responded efficaciously to PB, indicating age-dependent emergence of refractory neonatal seizures (P7 vs. P10), and were not associated with significant BDNF/ TrkB pathway activation. (f) Chemogenetic inactivation of the TrkB receptor in $\mathrm{P} 7$ pups resulted in a significant reduction in their postischemic seizure susceptibilities, supporting the role of BDNF/TrkB pathway activation in aggravation of neonatal seizures. (g) The developmental expression profiles demonstrated a significant decline in TrkB and PLC $\gamma 1$ expression driven by female pups versus a significant increase in KCC2 expression driven by male pups from P5 to P21. (h) Early in development, females showed significantly higher TrkB expression in the cortex, which may underlie the differences in LM antiseizure efficacy by sex. 
$L M$ functioned as a TrkB antagonist for endogenous BDNF after ischemia. The therapeutic potential of neurotrophin-based treatments for neurological diseases is an active field of preclinical research (37). In contrast to the role of neurotrophins in adulthood, the role of neurotrophin-based interventions in the developing neonatal brain is only recently emerging $(38,39)$. BDNF/TrkB signaling is age dependent: BDNF robustly activates $\operatorname{TrkB}$ in the neonatal rodent brain but is attenuated in the adult rodent brain (40). In the human frontal cortex, BDNF expression may gradually decrease with age (41). After BDNF binding, TrkB forms a homodimer and autophosphorylates Tyr residues in its intracellular domain (42). The phosphorylation of the TrkB homodimer residues initiates multiple downstream signal transduction pathways that affect neuronal survival, synaptogenesis, dendritic structure, and activity-dependent synaptic plasticity in a cell type-specific manner (43-45). Further, these signaling pathways are dependent upon the time course of BDNF/TrkB activation, in that acute versus chronic activation of $\mathrm{BDNF} / \operatorname{TrkB}$ is associated with divergent outcomes (44, $46,47)$. The complexity of BDNF function is apparent in its role as a selective regulator of gene expression via its modulation of RNA-binding proteins and microRNAs (reviewed in ref. 48). Additionally, both the human and rodent $B D N F$ genes consist of 9 exons, each with their own promoters, resulting in at least 10 different transcripts (reviewed in ref. 49). These alternative $B d n f$ transcripts undergo unique temporal and spatial expression that allow different factors, such as hypoxic response elements, to regulate BDNF signaling in a cell type-specific and circuit-specific manner $(38,50)$. The overexpression of a cleavage-resistant precursor of $\mathrm{BDNF}$ (pro-BDNF) has been demonstrated to reduce KCC2 protein expression via the $\mathrm{p} 75$ neurotrophin receptor (51), further highlighting the complexity of $\mathrm{BDNF} / \mathrm{TrkB}$ signaling in health and disease.

Downstream activation of the TrkB/PLC $\gamma 1$ pathway results in KCC2 hypofunction $(11,12,52)$. In an adult model of limbic epilepsy, prevention of PLC $\gamma 1$ pathway activation prevented epileptogenesis (53, 54). Further, AAV-Cre-mediated reduction of KCC2 in the CA1 and dentate gyrus of the adult mouse hippocampus resulted in some of the core phenotypes of medial temporal lobe epilepsy, such as spontaneous seizures, gliosis, and neuronal loss (55). These results from adult mouse models of epilepsy, with the prevalence of pathogenic KCC2 mutations in human epilepsy (56), support the critical role of KCC2 and pathways that promote its hypofunction in epilepsy.

Previous work in the neonatal brain has demonstrated the importance of preventing BDNF/TrkB activation after excitotoxic injury when using a small-molecule TrkB antagonist ANA12 (ref. 5 and see schematic, Figure 7). ANA12 prevented the activation of the TrkB/PLC $\gamma 1$ pathway, reversed postischemic KCC 2 hypofunction, and rescued P7 PB-refractory seizures $(2,5)$. In this same neonatal mouse model, the BDNF loop II mimetic LM also prevented BDNF-mediated TrkB/PLC $\gamma 1$ pathway activation and KCC2 hypofunction. In conjunction with the proposed binding of LM to TrkB (14), and the observation that LM intervention was efficacious, our data suggested that the presence and subsequent binding of LM to TrkB also prevented the cascade of endogenous postischemic BDNF/TrkB signaling, similar to the TrkB antagonist ANA12.

The 0.25 - and $2.5-\mathrm{mg} / \mathrm{kg}$ doses of LM, when given after ischemia, both rescued PB-refractory seizures in this study. In contrast, the low-dose of LM $(0.025 \mathrm{mg} / \mathrm{kg})$, when given after ischemia, resulted in a significantly aggravated first-hour baseline seizure burden compared with the ligate+PB group. These results may indicate that the lowest dose for LM tested in this study not only failed to rescue PB refractoriness, but also independently aggravated neonatal ischemic seizures. Therefore, higher doses of TrkB agonists may be required to have an effect on postischemic BDNF/TrkB activation in the neonatal brain $(2,5,15)$. The lowest dose of LM $(0.025 \mathrm{mg} / \mathrm{kg})$ was associated with an increase of p-KCC2-S940/KCC2 at 24 hours, but failed to acutely rescue PB refractoriness after ischemia. Future studies that assess the temporal dynamics of BDNF release in the neonatal brain after ischemia will provide valuable insights into the low-dose effects of LM on p-KCC2-S940/KCC2 at 24 hours.

TrkB/ERK1/2 pathway modulation by LM. LM binding to TrkB led to the activation of the TrkB/ ERK1/2 pathway, similar to previous reports from in vitro studies that peptide mimetics of loop I, III, or IV of BDNF can induce AKT and ERK phosphorylation $(57,58)$. TrkB activation also phosphorylates residue Y816 on its intracellular domain, permitting recruitment and activation of the PLC $\gamma 1$ pathway, which activates numerous pathways, including the $\mathrm{Ca}^{2+} /$ calmodulin-dependent kinases $(59,60)$. In this study, LM rescued the ischemia-induced phosphorylation of Y816 on TrkB, suggesting that the prevention of TrkB/ PLC $\gamma 1$ pathway-mediated KCC2 degradation occurred by LM binding to TrkB.

It has been shown that the use of HIOC in vivo protects retinas from excitotoxic retinal degeneration in a TrkB-dependent manner (16). Although both LM and HIOC significantly rescued PB refractoriness at P7, HIOC functioned differently from LM as it did not induce activation of the TrkB/ERK1/2 pathway. 


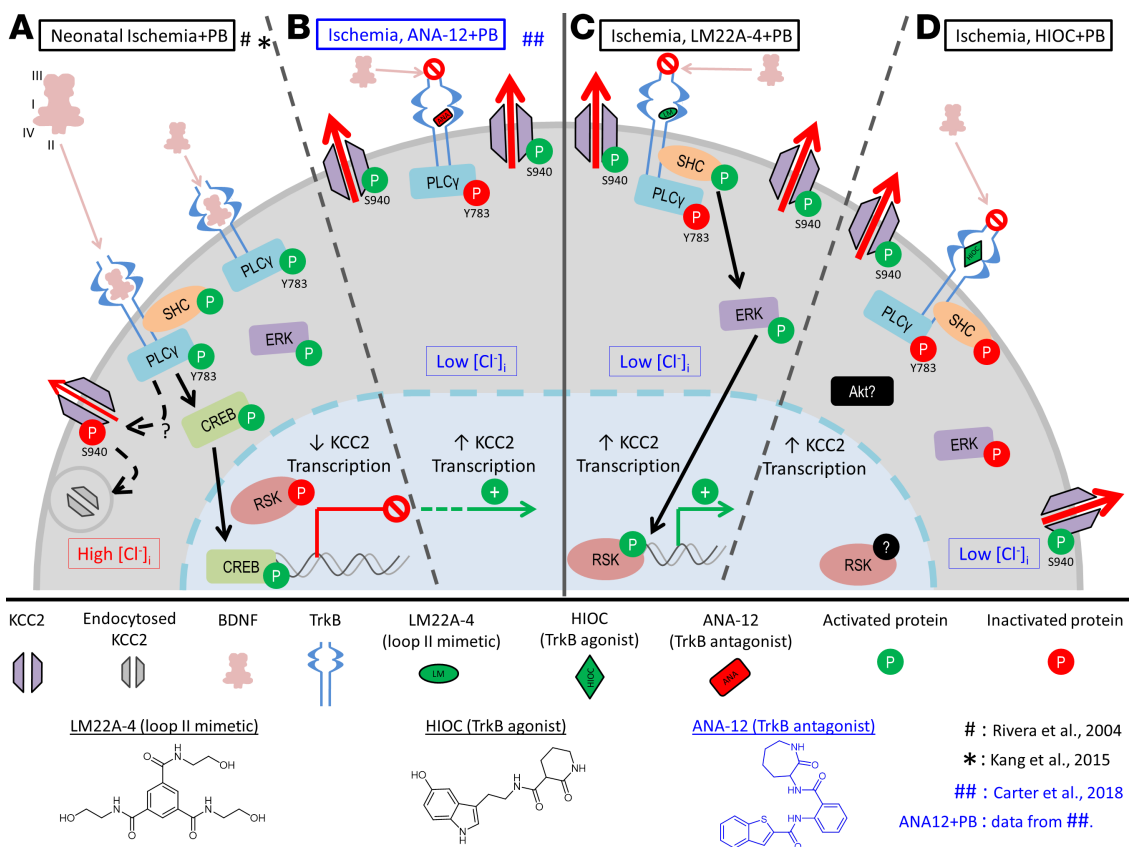

Figure 7. Summary schematic of TrkB signaling pathways after neonatal ischemia. (A) Endogenous postischemic BDNF release results in activation of the TrkB/PLC 1 pathway, thereby downregulating KCC2 expression (data summarized from refs. 12, 15) 24 hours after ischemia. (B) Treatment with the small-molecule TrkB antagonist ANA12 rescued postischemic TrkB/PLC $\gamma 1$ pathway activation-mediated KCC2 degradation (data summarized from ref. 5). (C) Intervention with LM22A-4, a TrkB partial agonist, also rescued TrkB/PLC $\gamma 1$ pathway activation, similar lyto ANA12, and activated the TrkB/ERK1/2 pathway instead. (D) Treatment with full TrkB agonist HIOC replicated the LM findings and rescued TrkB/PLC $\gamma 1$ pathway activation but did not activate the TrkB/ERK1/2 pathway, indicating that TrkB site-specific engagement dictates downstream cascades.

These results indicate that TrkB agonists could prevent emergence of refractory seizures by preventing the pathological BDNF/TrkB/PLC $\gamma 1$ pathway activation in postischemic neonatal brains, thus functionally acting as antagonists.

The ERK1/2 and AKT pathways are known to promote neurogenesis through the Ras signaling cascade (60), which regulates a multitude of processes, including cell migration, differentiation, proliferation, and transcription $(61,62)$. Previous literature has demonstrated that LM promoted cell survival in a model of nonarteritic anterior ischemic optic neuropathy (63). Supporting these results, the pre-LM group showed a significant increase in p-ERK1/2-T202/Y204 expression. Intervention with LM was shown to have beneficial long-term effects in vivo, particularly in rescuing adult posttraumatic cortical epileptogenesis in a TrkB-dependent manner (64), supporting the findings reported in this study.

Evidence for BDNF hyperactivity in neurological disorders. In autism, a severe neurodevelopmental disorder with pathogenesis that occurs during the neonatal period, the early hyperactivity of BDNF may play an important role because high BDNF levels have been reported in neonatal blood samples from children with autistic spectrum disorders $(65,66)$. Valproic acid (VPA) exposure during pregnancy increases the risk of congenital malformations and autism (67-69). VPA administration to pregnant rodents during the second week of gestation is a well-investigated model of autism (70). The VPA model of autism has demonstrated increased BDNF (70), increased neuronal intracellular chloride concentration (71), and a disruption of the GABA developmental sequence $(71,72)$. Research using a preclinical model of Fragile X syndrome, an inherited form of intellectual disability and autism spectrum disorder, administered LM to neonatal Fmr1-KO mice and rescued cell type-specific cortical developmental alterations (73). These studies suggest that TrkB agonists have a potential role in neurodevelopmental disorders with high levels of BDNF such as autism.

TrkB agonists can differentially activate selective downstream pathways, and TrkB/PLC $\gamma 1$ activation is complex. This concept is already evident in recent literature that has shown evidence of TrkB receptor activation and signaling without dimerization, suggesting that TrkB can under certain conditions exist and function as a monomeric receptor at the plasma membrane (74). Recent studies examining plasma 
membrane diffusion kinetics have demonstrated that up to $20 \%$ of the Trk family may exist as dimers or oligomers before neurotrophin binding (75), although their function remains a topic of debate (76). These observations warrant further investigation of the complexities involved in neurotrophin-based signaling in vivo. One specific example of the in vivo complexity of BDNF/TrkB signaling is in the superoxide dismutase 1 mouse model of familial ALS (77). In this model, the complete deletion of TrkB in motor neurons in vivo slowed the progression of the disease and permitted the maintenance of motor function (77). Another indication of the complexity of BDNF/TrkB signaling is in vitro studies that have identified that an increase in BDNF renders motor neurons more susceptible to excitotoxic insults in a TrkB-dependent manner; however, not all motor neurons were protected by blocking TrkB (78).

Sexually dimorphic developmental profiles of TrkB and KCC2. TrkB signaling is known to be sexually dimorphic in a region-specific manner (79-81). In $\mathrm{BDNF}^{+/-}$mice, greater levels of $\mathrm{p}$-TrkB-Y705 activation were shown in the frontal cortex and striatum of male mice 10-16 weeks of age compared with females. These changes in TrkB phosphorylation state were accompanied by concomitant increases in ERK1/2 phosphorylation (79), suggesting a differential signaling mechanism that was sexually dimorphic. The developmental expression of KCC2 is also known to increase with age, and this developmental profile is sex dependent: female pups express higher levels than male pups $(15,82,83)$. Similarly, rodent models have demonstrated higher female expression of KCC2 mRNA compared with age-matched males in the substantia nigra and mediobasal hypothalamus $(82,84)$.

Conclusion. The findings reported here demonstrated that the BDNF loop II mimetic LM and full TrkB agonists HIOC and DG significantly rescued $\mathrm{PB}$ refractoriness and prevented postischemic degradation of KCC2. Sex-dependent differences in developmental profiles for TrkB and KCC2 were identified that may underlie the significant sex-dependent variance in efficacy noted for LM and the full TrkB agonists. Additionally, the TrkB receptor plays a unique role in postischemic seizure susceptibility in the neonatal brain as shown using chemogenetic techniques. In summary, results of this study and previous results from a small-molecule TrkB antagonist ANA12 $(2,5)$ indicate that refractory seizures after neonatal ischemia can be rescued acutely by TrkB antagonists and agonists. These findings indicate that under neonatal ischemic conditions, the TrkB agonists investigated here pharmacologically acted similarly to TrkB antagonists by preventing cascades associated with endogenous BDNF/TrkB pathway activation, providing potentially novel insights into postischemic pathophysiology in immature brains. This highlights the crucial role of the TrkB receptor in neonatal seizure susceptibility and emergence of refractory seizures.

\section{Methods}

Experimental design. Litters of CD-1 pups were purchased from Charles River Laboratories, Inc. Pups with dams (litter size $=10$ ) were delivered on P3 and allowed to acclimate. Food and water were provided ad libitum. Doses for LM used in vivo and in vitro were determined from previously described in vitro pharmacokinetics (14). Pups of both sexes at P7 and P10 (see Supplemental Table 1 for sample sizes) were i.p. administered (Figure 1A) $0.25 \mathrm{mg} / \mathrm{kg}$ LM dissolved in isotonic PBS as 2 treatment groups: (a) 1 dose 2 hours before ligation (pre-LM) and (b) immediately following ligation (post-LM). After 1 hour of baseline EEG recording, pups were given a loading dose of $\mathrm{PB}(25 \mathrm{mg} / \mathrm{kg}$, i.p.). Doses for HIOC used in vivo and in vitro were determined from previously described in vitro pharmacokinetics (16). Pups of both sexes at P7 were i.p. administered the full agonist selective for TrkB $5 \mathrm{mg} / \mathrm{kg}$ HIOC dissolved in 95\%/5\% isotonic PBS/DMSO as 2 treatment groups: (a) 1 dose 2 hours before ligation (pre-HIOC) and (b) immediately following ligation (post-HIOC). Pups of both sexes at P7 were i.p. administered $5 \mathrm{mg} / \mathrm{kg}$ the full agonist selective for TrkB, DG, dissolved in 95\%/5\% isotonic PBS/DMSO as 2 treatment groups: (a) 1 dose 2 hours before ligation (pre-DG) and (b) immediately following ligation (post-DG). During the course of the experiments, DG became commercially unavailable, which is reflected in the smaller sample size for the DG-treated groups. We have previously shown that the 5\% DMSO used as a vehicle for drug administration did not have any antiseizure effect and did not alter baseline seizure burdens (2).

To run analysis for drug efficacies, data for naive male and female pups were pooled across all treatment groups for P7. The pups in the ligate+PB group were pooled from the LM, LM $0.025 \mathrm{mg} / \mathrm{kg}, \mathrm{LM}$ $2.5 \mathrm{mg} / \mathrm{kg}$, and HIOC treatment age-matched littermates. Every litter used in this study included pups randomly assigned to the naive control and ligate $+\mathrm{PB}$ treatment groups to ensure that every litter was represented in the naive and ligate+PB baseline data sets for EEG and Western blot. Experiments for DG were performed as an additional positive control, and the ligate+PB group was pooled for LM, LM $2.5 \mathrm{mg} / \mathrm{kg}$, and DG treatment littermates (see Supplemental Table 1 for sample size details). 
Surgery protocol for carotid ligation and EEG electrode implantation. At P7 or P10, pups underwent permanent unilateral ligation of the right common carotid artery without transection using $6 / 0$ silk sutures (Fine Science Tools) under isoflurane anesthesia (Henry Schein). The skin was closed with 6/0 monofilament nylon (Covidien), and lidocaine was applied as a local anesthetic. Pups were then implanted with 3 subdermal EEG electrodes (SWE-L25, Ives EEG Solutions): 1 recording and 1 reference overlaying the left/right parietal cortex, and 1 ground overlaying the rostrum $(15,23)$. The electrodes were fixed in place using cyanoacrylate adhesive (KrazyGlue). Pups were tethered to a preamplifier inside the recording chamber and allowed to recover from anesthesia. Video EEG was recorded continuously for 2 hours in a chamber maintained at $37^{\circ} \mathrm{C}$ with isothermal pads. At the end of recording, the electrodes were removed and pups were returned to the dam.

EEG recordings and analyses. EEG recordings were acquired using Sirenia Acquisition (version 1.6.4, Pinnacle Technology, Inc.) with synchronized video recording. Data were recorded with $400 \mathrm{~Hz}$ sampling rate that had a preamplifier gain of 100 , at $0.5-50 \mathrm{~Hz}$ to remove ambient noise. The EEG data were binned into 10-second epochs for manual scoring. Seizures were defined as electrographic events consisting of rhythmic spikes of high amplitude, diffuse peak frequency equal to or greater than $7-8 \mathrm{~Hz}$ lasting 6 seconds or longer, similar to previous studies $(5,23)$. Similar to previously published protocols in this model, short-duration burst activity lasting less than 6 seconds was not included in seizure burden calculations. Seizure suppression was calculated as ([second-hour seizure burden - first-hour seizure burden]/ first-hour seizure burden) $\times 100$.

EEG power analysis. Four P7 pups of each sex in each treatment group were randomly chosen for EEG power analysis. EEG power was obtained with Sirenia Sleep (version 1.7.10, Pinnacle Technology Inc.). EEG spectral power from 0.5 to $50 \mathrm{~Hz}$ was acquired for each 10-second epoch of recording after automated fast Fourier transformation. Data from EEG artifacts were excluded from these analyses. Total EEG power was calculated as ([first-hour power - second-hour power]/first-hour power) $\times 100$.

P7 ligation in C57BL/6 knockin TrkB ${ }^{F 616 A}$ pups. TrkB ${ }^{F 616 A}$ breeding pairs (JAX stock 022363, developed by the David Ginty lab, ref. 85) were obtained courtesy of Richard Huganir (Johns Hopkins University). To investigate the effect of chemogenetically induced deficits in neonatal TrkB signaling in vivo, P7 pups with TrkB ${ }^{F 616 A}$-knockin alleles $\left(\mathrm{F} 616 \mathrm{~A}^{+/+}\right)(85)$ received the kinase inhibitor $1 \mathrm{NMPP} 1$ from P0 to P7 via transmammary route with the dam receiving the chemogenetic agent in drinking water $(10 \%$ Tween-20 and 80 $\mu \mathrm{M} 1 \mathrm{NMPP} 1$ in drinking water). $\mathrm{P} 7 \mathrm{WT}^{-/-}$and $\mathrm{F} 616 \mathrm{~A}^{+/+}$littermates with 1NMPP1 treatment underwent unilateral carotid ligation and subsequent video EEG at P7 as previously described.

Western blotting. All animals were anesthetized with $0.1 \mathrm{~mL}$ of $90 \mathrm{mg} / \mathrm{mL}$ chloral hydrate (i.p.) 24 hours after ligation. Pups were then transcardially perfused with ice-cold PBS followed by $1 \mathrm{~mL}$ of $1 \times$ HALT protease inhibitor cocktail in PBS (Thermo Fisher Scientific, 78430). The whole brains were removed, the cerebellum was discarded, and the left and right hemispheres were separated. For the developmental series, brains were further microdissected into cortex, hippocampus, and deep gray matter and stored at $-80^{\circ} \mathrm{C}$ until further processing. Homogenized brain tissue was suspended in T-PER cell lysis buffer containing $1 \times$ HALT protease inhibitor cocktail. Protein concentration was measured using the Bradford protein assay at $570 \mathrm{~nm}$. Then, $25 \mu \mathrm{g}$ of total protein ( $60 \mu \mathrm{g}$ for PLC $\gamma 1)$ in $20 \mu \mathrm{L}$ was run on $4 \%$ to $20 \%$ Tris-glycine gels (Thermo Fisher Scientific, XP04205BOX) for 120 minutes at $130 \mathrm{~V}$. Gels were transferred overnight at $20 \mathrm{~V}$ onto nitrocellulose membranes. Membranes were blocked in blocking buffer for 1 hour (Rockland, MB-070). Membranes underwent 6-hour incubation with primary antibodies: mouse $\alpha$-KCC2 (1:1000, Aviva Systems Biology, OASE00240), rabbit $\alpha$-phospho-KCC2 (S940) (1:1000, Aviva Systems Biology, OAPC00188), mouse $\alpha$-TrkB (1:1000, BD Biosciences, 610102), rabbit $\alpha$-phospho-TrkB (T816) (1:500, MilliporeSigma, ABN1381), mouse $\alpha$-PLC $\gamma 1$ (1:1000, Thermo Fisher Scientific, LF-MA0050), rabbit $\alpha$-phospho-PLC $\gamma 1$ (Y783) (1:1000, Cell Signaling Technology, 2821S), rabbit $\alpha$-Erk1/2 (1:1000, Cell Signaling Technology, 4695), rabbit $\alpha$-phospho-Erk1/2 (1:1000, Cell Signaling Technology, 377), and mouse $\alpha-\beta$-actin (1:10,000, Li-Cor Biosciences, 926-42212). Membranes were then incubated with fluorescent secondary antibodies (1:5000, Li-Cor Biosciences, 926-68020 and 925-32211; for antibody Research Resource Identifiers, see Supplemental Table 2). Blots were visualized on the Odyssey infrared imagining system 2.1 (Li-Cor Biosciences). Optical density for each protein band was normalized to $\beta$-actin in the same lane.

Surface protein separation by ultracentrifugation. One-millimeter coronal slices were obtained from P7 mouse brains and were allowed to recover for 45 minutes at $34^{\circ} \mathrm{C}$ with oxygenation $95 \% / 5 \% \mathrm{O}_{2} / \mathrm{CO}_{2}$. After recovery, slices were incubated with TrkB agonists LM22A-4 and HIOC at $34^{\circ} \mathrm{C}$ with oxygenation. 
Slices were placed in lysis buffer TPER with HALT protease and phosphatase inhibitors and homogenized. After a 30-minute incubation on ice, protein lysates were ultracentrifuged at 210,000 $\mathrm{g}$ (TLA-120.2 rotor, Beckman Coulter Life Sciences), and supernatant was collected as a cytosolic sample. Pellets were resuspended and ultracentrifuged; supernatant was discarded. Pellets were resuspended and collected as membrane components. Membrane and cytosolic components underwent Bradford analysis and Western blotting for protein quantification. Plasma membrane sample proteins were normalized to caveolin-1 (TrkB) or TfR (KCC2). Cytosolic sample proteins were normalized to $\beta$-actin.

Statistics. All statistical analyses were performed in Prism 7 (GraphPad). Percentage seizure suppression by sex, seizure burdens, ictal events, ictal durations, and protein expression levels quantified from P5 to P21 were analyzed using 2-way ANOVA, and post hoc corrections were made using Tukey's test. Percentage of seizure suppression and all Western blot data at P7 and P10 for drug efficacies were analyzed using 1-way ANOVA, and post hoc corrections were made using Holm-Šídák test. For comparisons between ipsilateral and contralateral hemispheres within groups at P7 and P10, as well as comparisons of baseline seizure burdens between developmental ages P7 and P10, 2-tailed $t$ tests were performed. Significance of correlations between the percentage of EEG spectral power suppression and percentage of seizure burden suppression across treatment groups were determined using Spearman's 2 -tailed nonparametric test. An $\alpha$ of $P$ less than 0.05 was considered significant. Data were plotted as box-and-whisker plots demonstrating minima, maxima, and median with quartiles. Dot plots for developmental protein expression show the mean \pm 1 SEM.

Study approval. All experiments were done in compliance with the Johns Hopkins University Committee on the Ethics of Animal Experiments (permit A3272-01) and approved by the Animal Care and Use Committee of Johns Hopkins.

\section{Author contributions}

SDK designed the research. PAK, BJS, BMC, and SDK performed the research. PAK, BJS, BMC, and SDK acquired data. PAK, BJS, and SDK analyzed data. PAK, BJS, and SDK wrote the paper.

\section{Acknowledgments}

This work was supported by the Eunice Kennedy Shriver National Institute of Child Health and Human Development of the NIH under award number R01HD090884 (to SDK). The content is solely the responsibility of the authors and does not necessarily represent the official views of the NIH.

Address correspondence to: Shilpa D. Kadam, Hugo W. Moser Research Institute at Kennedy Krieger, Johns Hopkins University School of Medicine, 707 North Broadway, 400H, Baltimore, Maryland 21205, USA. Phone: 443.923.2688; Email: kadam@kennedykrieger.org or skadam1@jh.edu.

1. Aloyz R, Fawcett JP, Kaplan DR, Murphy RA, Miller FD. Activity-dependent activation of TrkB neurotrophin receptors in the adult CNS. Learn Mem. 1999;6(3):216-231

2. Kang SK, Johnston MV, Kadam SD. Acute TrkB inhibition rescues phenobarbital-resistant seizures in a mouse model of neonatal ischemia. Eur J Neurosci. 2015;42(10):2792-2804.

3. He XP, Minichiello L, Klein R, McNamara JO. Immunohistochemical evidence of seizure-induced activation of TrkB receptors in the mossy fiber pathway of adult mouse hippocampus. J Neurosci. 2002;22(17):7502-7508.

4. Kowiański P, Lietzau G, Czuba E, Waśkow M, Steliga A, Moryś J. BDNF: a key factor with multipotent impact on brain signaling and synaptic plasticity. Cell Mol Neurobiol. 2018;38(3):579-593.

5. Carter BM, Sullivan BJ, Landers JR, Kadam SD. Dose-dependent reversal of KCC2 hypofunction and phenobarbital-resistant neonatal seizures by ANA12. Sci Rep. 2018;8(1):11987.

6. Lee HH, Walker JA, Williams JR, Goodier RJ, Payne JA, Moss SJ. Direct protein kinase C-dependent phosphorylation regulates the cell surface stability and activity of the potassium chloride cotransporter KCC2. J Biol Chem. 2007;282(41):29777-29784.

7. Lee HH, Deeb TZ, Walker JA, Davies PA, Moss SJ. NMDA receptor activity downregulates KCC2 resulting in depolarizing GABAA receptor-mediated currents. Nat Neurosci. 2011;14(6):736-743.

8. Ben-Ari Y, Khalilov I, Kahle KT, Cherubini E. The GABA excitatory/inhibitory shift in brain maturation and neurological disorders. Neuroscientist. 2012;18(5):467-486.

9. Sedmak G, et al. Developmental expression patterns of KCC2 and functionally associated molecules in the human brain. Cereb Cortex. 2016;26(12):4574-4589.

10. Hyde TM, et al. Expression of GABA signaling molecules KCC2, NKCC1, and GAD1 in cortical development and schizophrenia. JNeurosci. 2011;31(30):11088-11095.

11. Rivera C, et al. BDNF-induced TrkB activation down-regulates the $\mathrm{K}^{+}-\mathrm{Cl}^{-}$cotransporter $\mathrm{KCC}$ and impairs neuronal $\mathrm{Cl}^{-}$extru- 
sion. J Cell Biol. 2002;159(5):747-752.

12. Rivera C, et al. Mechanism of activity-dependent downregulation of the neuron-specific K-Cl cotransporter KCC2. J Neurosci. 2004;24(19):4683-4691.

13. Löscher W, Rogawski MA. How theories evolved concerning the mechanism of action of barbiturates. Epilepsia. 2012;53(suppl 8):12-25.

14. Massa SM, et al. Small molecule BDNF mimetics activate TrkB signaling and prevent neuronal degeneration in rodents. $J$ Clin Invest. 2010;120(5):1774-1785.

15. Kang SK, Markowitz GJ, Kim ST, Johnston MV, Kadam SD. Age- and sex-dependent susceptibility to phenobarbital-resistant neonatal seizures: role of chloride co-transporters. Front Cell Neurosci. 2015;9:173.

16. Shen J, et al. N-acetyl serotonin derivatives as potent neuroprotectants for retinas. Proc Natl Acad Sci U S A. 2012;109(9):3540-3545.

17. Jang SW, et al. Deoxygedunin, a natural product with potent neurotrophic activity in mice. PLoS One. 2010;5(7):e11528.

18. Setterholm NA, McDonald FE, Boatright JH, Iuvone PM. Gram-scale, chemoselective synthesis of $N$-[2-(5-hydroxy-1H-indol-3yl)ethyl]-2-oxopiperidine-3-carboxamide (HIOC). Tetrahedron Lett. 2015;56(23):3413-3415.

19. Croll SD, Ip NY, Lindsay RM, Wiegand SJ. Expression of BDNF and trkB as a function of age and cognitive performance. Brain Res. 1998;812(1-2):200-208.

20. Rage F, Silhol M, Binamé F, Arancibia S, Tapia-Arancibia L. Effect of aging on the expression of BDNF and TrkB isoforms in rat pituitary. Neurobiol Aging. 2007;28(7):1088-1098.

21. Silhol M, Bonnichon V, Rage F, Tapia-Arancibia L. Age-related changes in brain-derived neurotrophic factor and tyrosine kinase receptor isoforms in the hippocampus and hypothalamus in male rats. Neuroscience. 2005;132(3):613-624.

22. Webster MJ, Herman MM, Kleinman JE, Shannon Weickert C. BDNF and trkB mRNA expression in the hippocampus and temporal cortex during the human lifespan. Gene Expr Patterns. 2006;6(8):941-951.

23. Kharod SC, Carter BM, Kadam SD. Pharmaco-resistant neonatal seizures: critical mechanistic insights from a chemoconvulsant model. Dev Neurobiol. 2018;78(11):1117-1130.

24. Kipnis PA, Sullivan BJ, Kadam SD. Sex-dependent signaling pathways underlying seizure susceptibility and the role of chloride cotransporters. Cells. 2019;8(5):E448

25. Galanopoulou AS. Dissociated gender-specific effects of recurrent seizures on GABA signaling in CA1 pyramidal neurons: role of GABA(A) receptors. J Neurosci. 2008;28(7):1557-1567.

26. Calabrese EJ, Baldwin LA. U-shaped dose-responses in biology, toxicology, and public health. Annu Rev Public Health 2001;22:15-33.

27. Dzhala VI, et al. NKCC1 transporter facilitates seizures in the developing brain. Nat Med. 2005;11(11):1205-1213.

28. Sato SM, Woolley CS. Acute inhibition of neurosteroid estrogen synthesis suppresses status epilepticus in an animal model. Elife. 2016;5:e12917.

29. Zayachkivsky A, Lehmkuhle MJ, Ekstrand JJ, Dudek FE. Ischemic injury suppresses hypoxia-induced electrographic seizures and the background EEG in a rat model of perinatal hypoxic-ischemic encephalopathy. J Neurophysiol. 2015;114(5):2753-2763.

30. Rennie JM, et al. Characterisation of neonatal seizures and their treatment using continuous EEG monitoring: a multicentre experience. Arch Dis Child Fetal Neonatal Ed. 2019;104(5):F493-F501.

31. Sheldon RA, Sedik C, Ferriero DM. Strain-related brain injury in neonatal mice subjected to hypoxia-ischemia. Brain Res. 1998;810(1-2):114-122.

32. Sheldon RA, Windsor C, Ferriero DM. Strain-related differences in mouse neonatal hypoxia-ischemia. Dev Neurosci. 2018;40(5-6):490-496.

33. Kang SK, Hawkins NA, Kearney JA. C57BL/6J and C57BL/6N substrains differentially influence phenotype severity in the Scn1 $a^{+/-}$mouse model of Dravet syndrome. Epilepsia Open. 2019;4(1):164-169.

34. de la Tremblaye PB, Benoit SM, Schock S, Plamondon H. CRHR1 exacerbates the glial inflammatory response and alters $\mathrm{BDNF} / \mathrm{TrkB} / \mathrm{pCREB}$ signaling in a rat model of global cerebral ischemia: implications for neuroprotection and cognitive recovery. Prog Neuropsychopharmacol Biol Psychiatry. 2017;79(pt B):234-248.

35. He XP, Pan E, Sciarretta C, Minichiello L, McNamara JO. Disruption of TrkB-mediated phospholipase Cgamma signaling inhibits limbic epileptogenesis. J Neurosci. 2010;30(18):6188-6196.

36. Almeida RD, et al. Neuroprotection by BDNF against glutamate-induced apoptotic cell death is mediated by ERK and PI3-kinase pathways. Cell Death Differ. 2005;12(10):1329-1343.

37. Longo FM, Massa SM. Small-molecule modulation of neurotrophin receptors: a strategy for the treatment of neurological disease. Nat Rev Drug Discov. 2013;12(7):507-525.

38. Hempstead BL. Brain-derived neurotrophic factor: three ligands, many actions. Trans Am Clin Climatol Assoc. 2015;126:9-19.

39. Subedi L, et al. Plasma brain-derived neurotrophic factor levels in newborn infants with neonatal abstinence syndrome. Front Pediatr. 2017;5:238.

40. Knüsel B, Rabin SJ, Hefti F, Kaplan DR. Regulated neurotrophin receptor responsiveness during neuronal migrationand early differentiation. J Neurosci. 1994;14(3 pt 2):1542-1554.

41. Oh H, Lewis DA, Sibille E. The role of BDNF in age-dependent changes of excitatory and inhibitory synaptic markers in the human prefrontal cortex. Neuropsychopharmacology. 2016;41(13):3080-3091.

42. Deinhardt K, Chao MV. Trk receptors. Handb Exp Pharmacol. 2014;220:103-119.

43. Chao MV. Neurotrophins and their receptors: a convergence point for many signalling pathways. Nat Rev Neurosci. 2003;4(4):299-309.

44. Gottmann K, Mittmann T, Lessmann V. BDNF signaling in the formation, maturation and plasticity of glutamatergic and GABAergic synapses. Exp Brain Res. 2009;199(3-4):203-234.

45. Huang EJ, Reichardt LF. Trk receptors: roles in neuronal signal transduction. Annu Rev Biochem. 2003;72:609-642.

46. Binder DK, Croll SD, Gall CM, Scharfman HE. BDNF and epilepsy: too much of a good thing? Trends Neurosci. 2001;24(1):47-53

47. McNamara JO, Scharfman HE. Temporal lobe epilepsy and the BDNF receptor, TrkB. In: Noebels JL, et al, eds. Jasper's Basic Mechanisms of the Epilepsies. Bethesda, Maryland, USA: National Center for Biotechnology Information; 2012.

48. Ruiz CR, Shi J, Meffert MK. Transcript specificity in BDNF-regulated protein synthesis. Neuropharmacology. 2014;76(pt C):657-663. 
49. Chen KW, Chen L. Epigenetic regulation of BDNF gene during development and diseases. Int J Mol Sci. 2017; 18(3):E571.

50. Maynard KR, et al. Functional role of BDNF production from unique promoters in aggression and serotonin signaling. Neuropsychopharmacology. 2016;41(8):1943-1955

51. Riffault B, et al. Pro-brain-derived neurotrophic factor (proBDNF)-mediated p75NTR activation promotes depolarizing actions of GABA and increases susceptibility to epileptic seizures. Cereb Cortex. 2018;28(2):510-527.

52. Nardou R, et al. Neuronal chloride accumulation and excitatory GABA underlie aggravation of neonatal epileptiform activities by phenobarbital. Brain. 2011;134(pt 4):987-1002.

53. Gu B, Huang YZ, He XP, Joshi RB, Jang W, McNamara JO. A peptide uncoupling BDNF receptor TrkB from phospholipase C $\gamma 1$ prevents epilepsy induced by status epilepticus. Neuron. 2015;88(3):484-491.

54. He XP, Pan E, Sciarretta C, Minichiello L, McNamara JO. Disruption of TrkB-mediated phospholipase C $\gamma$ signaling inhibits limbic epileptogenesis. J Neurosci. 2010;30(18):6188-6196.

55. Kelley MR, et al. Locally reducing KCC2 activity in the hippocampus is sufficient to induce temporal lobe epilepsy. EBioMedicine. 2018;32:62-71.

56. Duy PQ, David WB, Kahle KT. Identification of KCC2 mutations in human epilepsy suggests strategies for therapeutic transporter modulation. Front Cell Neurosci. 2019;13:515.

57. Fobian K, Owczarek S, Budtz C, Bock E, Berezin V, Pedersen MV. Peptides derived from the solvent-exposed loops 3 and 4 of BDNF bind TrkB and p75(NTR) receptors and stimulate neurite outgrowth and survival. J Neurosci Res. 2010;88(6):1170-1181.

58. Gudasheva TA, Povarnina P, Logvinov IO, Antipova TA, Seredenin SB. Mimetics of brain-derived neurotrophic factor loops 1 and 4 are active in a model of ischemic stroke in rats. Drug Des Devel Ther. 2016;10:3545-3553.

59. Gärtner A, et al. Hippocampal long-term potentiation is supported by presynaptic and postsynaptic tyrosine receptor kinase B-mediated phospholipase C $\gamma$ signaling. J Neurosci. 2006;26(13):3496-3504.

60. Minichiello L. TrkB signalling pathways in LTP and learning. Nat Rev Neurosci. 2009;10(12):850-860.

61. Han BH, Holtzman DM. BDNF protects the neonatal brain from hypoxic-ischemic injury in vivo via the ERK pathway. $J$ Neurosci. 2000;20(15):5775-5781.

62. Roskoski R. ERK1/2 MAP kinases: structure, function, and regulation. Pharmacol Res. 2012;66(2):105-143.

63. Ali Shariati M, et al. A small molecule TrkB neurotrophin receptor partial agonist as possible treatment for experimental nonarteritic anterior ischemic optic neuropathy. Curr Eye Res. 2018;43(12):1489-1499.

64. Gu F, Parada I, Yang T, Longo FM, Prince DA. Partial TrkB receptor activation suppresses cortical epileptogenesis through actions on parvalbumin interneurons. Neurobiol Dis. 2018;113:45-58.

65. Nelson KB, et al. Neuropeptides and neurotrophins in neonatal blood of children with autism or mental retardation. Ann Neurol. 2001;49(5):597-606.

66. Tsai SJ. Is autism caused by early hyperactivity of brain-derived neurotrophic factor? Med Hypotheses. 2005;65(1):79-82.

67. Bromley RL, Mawer G, Clayton-Smith J, Baker GA, Liverpool Manchester Neurodevelopment Group. Autism spectrum disorders following in utero exposure to antiepileptic drugs. Neurology. 2008;71(23):1923-1924.

68. Bromley RL, et al. The prevalence of neurodevelopmental disorders in children prenatally exposed to antiepileptic drugs. $J$ Neurol Neurosurg Psychiatry. 2013;84(6):637-643.

69. Williams G, King J, Cunningham M, Stephan M, Kerr B, Hersh JH. Fetal valproate syndrome and autism: additional evidence of an association. Dev Med Child Neurol. 2001;43(3):202-206.

70. Almeida LE, Roby CD, Krueger BK. Increased BDNF expression in fetal brain in the valproic acid model of autism. Mol Cell Neurosci. 2014;59:57-62.

71. Tyzio R, et al. Oxytocin-mediated GABA inhibition during delivery attenuates autism pathogenesis in rodent offspring. Science. 2014;343(6171):675-679.

72. Cloarec R, et al. Pyramidal neuron growth and increased hippocampal volume during labor and birth in autism. Sci Adv 2019;5(1):eaav0394.

73. Nomura T, et al. Delayed maturation of fast-spiking interneurons is rectified by activation of the TrkB receptor in the mouse model of fragile X syndrome. J Neurosci. 2017;37(47):11298-11310.

74. Zahavi EE, et al. The receptor tyrosine kinase TrkB signals without dimerization at the plasma membrane. Sci Signal. 2018;11(529):eaao4006.

75. Marchetti L, et al. Ligand signature in the membrane dynamics of single TrkA receptor molecules. J Cell Sci. 2013;126(pt 19):4445-4456.

76. Maruyama IN. Mechanisms of activation of receptor tyrosine kinases: monomers or dimers. Cells. 2014;3(2):304-330

77. Zhai J, et al. The in vivo contribution of motor neuron TrkB receptors to mutant SOD1 motor neuron disease. Hum Mol Genet. 2011;20(21):4116-4131.

78. Hu P, Kalb RG. BDNF heightens the sensitivity of motor neurons to excitotoxic insults through activation of TrkB. JNeurochem. 2003;84(6):1421-1430.

79. Hill RA, van den Buuse M. Sex-dependent and region-specific changes in TrkB signaling in BDNF heterozygous mice. Brain Res. 2011;1384:51-60.

80. Scharfman HE, MacLusky NJ. Differential regulation of BDNF, synaptic plasticity and sprouting in the hippocampal mossy fiber pathway of male and female rats. Neuropharmacology. 2014;76(pt C):696-708.

81. Scharfman HE, Maclusky NJ. Similarities between actions of estrogen and BDNF in the hippocampus: coincidence or clue? Trends Neurosci. 2005;28(2):79-85.

82. Galanopoulou AS, Kyrozis A, Claudio OI, Stanton PK, Moshé SL. Sex-specific KCC2 expression and GABA(A) receptor function in rat substantia nigra. Exp Neurol. 2003;183(2):628-637.

83. Galanopoulou AS. Sex- and cell-type-specific patterns of GABAA receptor and estradiol-mediated signaling in the immature rat substantia nigra. Eur J Neurosci. 2006;23(9):2423-2430.

84. Perrot-Sinal TS, Sinal CJ, Reader JC, Speert DB, McCarthy MM. Sex differences in the chloride cotransporters, NKCC1 and KCC2, in the developing hypothalamus. J Neuroendocrinol. 2007;19(4):302-308.

85. Chen X, et al. A chemical-genetic approach to studying neurotrophin signaling. Neuron. 2005;46(1):13-21. 